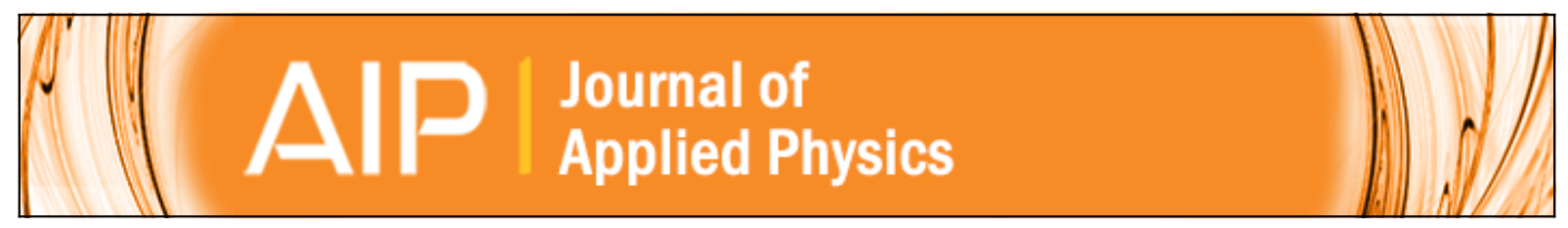

\title{
Modeling the electrical impedance response of ionic polymer transducers
}

Kevin M. Farinholt and Donald J. Leo

Citation: Journal of Applied Physics 104, 014512 (2008); doi: 10.1063/1.2952974

View online: http://dx.doi.org/10.1063/1.2952974

View Table of Contents: http://scitation.aip.org/content/aip/journal/jap/104/1?ver=pdfcov

Published by the AIP Publishing

\section{Articles you may be interested in}

A physics-based model of the electrical impedance of ionic polymer metal composites

J. Appl. Phys. 111, 124901 (2012); 10.1063/1.4729051

High surface area electrodes in ionic polymer transducers: Numerical and experimental investigations of the electro-chemical behavior

J. Appl. Phys. 109, 074509 (2011); 10.1063/1.3556751

Boundary layer charge dynamics in ionic liquid-ionic polymer transducers

J. Appl. Phys. 109, 014909 (2011); 10.1063/1.3524189

Improving an electret transducer by fully utilizing the implanted charge

Appl. Phys. Lett. 93, 122901 (2008); 10.1063/1.2985899

Modeling of electrochemomechanical response of ionic polymer-metal composites with various solvents J. Appl. Phys. 100, 064310 (2006); 10.1063/1.2221505

MIT LINCOLN LABORATORY CAREERS

Discover the satisfaction of innovation and service to the nation
- Space Control

- Air \& Missile Defense

- Communications Systems \& Cyber Security

- Intelligence, Surveillance and

Reconnaissance Systems

$$
\begin{aligned}
& \text { Electronics } \\
& \text { - Tactical Systems } \\
& \text { - Homeland } \\
& \text { Protection } \\
& \text { - Air Traffic Control }
\end{aligned}
$$$$
\text { Advanced }
$$

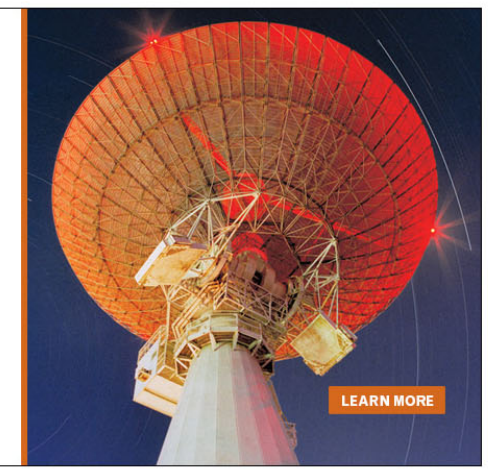




\title{
Modeling the electrical impedance response of ionic polymer transducers
}

\author{
Kevin M. Farinholt ${ }^{1, a)}$ and Donald J. Leo ${ }^{2}$ \\ ${ }^{1}$ The Engineering Institute, Los Alamos National Laboratory, Los Alamos, New Mexico 87545, USA \\ ${ }^{2}$ Center for Intelligent Material Systems and Structures, Virginia Tech, Blacksburg, Virginia 24061, USA
}

(Received 14 December 2007; accepted 3 May 2008; published online 15 July 2008)

\begin{abstract}
An analytical study is presented that investigates the electrical impedance response of the ionic polymer transducer. Experimental studies have shown that the electromechanical response of these active materials is highly dependent upon internal parameters such as neutralizing counterion, diluent, electrode treatment, as well as environmental factors such as ambient temperature. Further examination has shown that these variations are introduced predominantly through the polymer's ability to convert voltage into charge migration. This relationship can easily be represented by the polymer's electrical impedance as measured across the outer electrodes of the transducer. In the first half of this study an analytical model is developed which predicts the time and frequency domain characteristics of the electrical response of the ionic polymer transducer. Transport equations serve as the basis for this model, from which a series of relationships are developed to describe internal potential, internal charge density, as well as surface current. In the second half of this study several analytical studies are presented to understand the impact that internal parameters have on the polymer's electrical response, while providing a conceptual validation of the model. In addition to the analytical studies several experimental comparisons are made to further validate the model by examining how well the model predicts changes in temperature, viscosity and pretention within the ionic polymer transducer. (C) 2008 American Institute of Physics. [DOI: 10.1063/1.2952974]
\end{abstract}

\section{INTRODUCTION}

The ionic polymer transducer (IPT) represents a new class of active material that exhibits coupling between electrical, chemical, and mechanical domains. While these materials were initially recognized for their chemoelectric properties, some of the earliest work with the chemomechanical and electromechanical behaviors was conducted in the 1940s-1960s by Katchalsky, ${ }^{1}$ Kuhn et al., ${ }^{2,3}$ and Hamlen et $a{ }^{4}{ }^{4}$ Other researchers continued this work through the $1970 \mathrm{~s}$ (Refs. 5 and 6) and 1980s, ${ }^{7}$ however, it was not until the 1990s that large scale research efforts were focused on the ionic polymer as an electromechanical transducer. In 1992, Oguro et al. ${ }^{8}$ and Sadeghipour et al. ${ }^{9}$ presented work demonstrating that the ionic polymer could respond as either an electromechanical actuator ${ }^{8}$ or a sensor ${ }^{9}$ when suitably plated with conductive electrodes. From these initial studies and the results of Segalman et al., ${ }^{10}$ a considerable research effort has been focused on characterizing and modeling the electromechanical response of the ionic polymer transducer. The physical response of the ionic polymer transducer is that of an inherent bender. Under relatively low electric fields (1-10 $\mathrm{kV} / \mathrm{m}$ ) these materials exhibit large bending strains which can be tailored through changes in the polymer structure, ${ }^{11-13}$ fixed and mobile ion species, ${ }^{13-15}$ diluent properties ${ }^{15-17}$ and the electrode composition and structure. ${ }^{18}$ Each of these parameters has been experimentally shown to greatly influence both the actuation and sensing response of the ionic polymer transducer.

Over the years, numerous models have been proposed to describe the actuation and sensing properties of the ionic

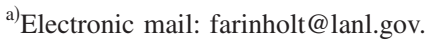

polymer bender. Each of these models can be thought to offer varying degrees of insight into the inner mechanisms at work within the IPT. The broadest class of models can be thought of as black box models, offering input-output relationships based upon curve fits of experimental data. This class of models began to emerge in the mid 1990s with work by Kanno et al., ${ }^{19,20}$ who addressed the actuation response of the IPT. This concept was further extended by Newbury and Leo, ${ }^{21-23}$ who developed a constitutive model that addressed both the sensing and actuation response of the IPT. The advantage of this work is that both sensing and actuation are modeled through a single, two-port representation that allows for geometric scaling of the transducer and offers suitable design capabilities for low frequency applications. In addition to this class of models, a series of more in depth models have emerged which try to understand the physics responsible for the sensing and actuation responses of the ionomer-based transducers. de Gennes et al. ${ }^{24}$ proposed a static model based upon the concept of irreversible thermodynamics, utilizing electric field, current, pressure gradient, and water flux as state variables in their model. Their model parameters are the electric conductivity of the material, hydraulic permeability, and a cross-coupling term that models transduction between water flow and the flow of charge. Tadokoro et $a .^{25}$ have proposed another model based upon ionic motion within the polymer. In the Tadokoro model, electromechanical transduction is attributed to the forces imposed upon the ions as they migrate through the polymer due to an imposition of electric field. Electrostatic interactions produced by ionic motion are also modeled, and interestingly they are able to predict the counter motion of ionic polymer benders that is sometimes exhibited when excited by a constant electric field. 
Beyond the grey box models of de Gennes et al. ${ }^{24}$ and Tadokoro et al., ${ }^{25}$ a separate class of models have been developed using a field approach based upon the micromechanics and electrostatics of the ionic polymer membrane. Nemat-Nasser et $_{\text {al. }}{ }^{26-28}$ have proposed that actuation occurs due to the uptake of water at the interface between the polymer and the metal electrode. ${ }^{26}$ In contrast to the work by de Gennes et al. ${ }^{24}$ and Tadokoro et al. ${ }^{25}$ the models by NematNasser et al. ${ }^{26,27}$ do not explicitly include water motion and hydraulic pressure gradients as state variables. An ionic cluster model of the base material provides the basis for modeling the electromechanical transduction between charge distribution and mechanical deformation. This work inspired later work by Xiao and Bhattacharya ${ }^{29}$ who constructed an elastoelectrochemical formulation which describes the actuation response of the IPT. They treat the polymer as a soft insulating material that exhibits elastic properties, while allowing ions to move freely throughout the medium. Their results generally support the work of Nemat-Nasser et $a l .,{ }^{26,27}$ but differ slightly in their predictions of ion accumulation near the polymer electrode. Farinholt and $\mathrm{Leo}^{14}$ also use the electrostatics approach to model the IPT, focusing on the charge sensing response of the IPT rather than the actuation response. This work relaxes the assumption of zero ionic flux and accurately predicts the time response for a step deformation in the tip of the ionic polymer transducer. Weiland and Leo ${ }^{30-32}$ also expand upon the analysis of Nemat-Nasser to consider the response of a single ion cluster within the ionomer membrane. Their work considers both electrically and mechanically induced excitations upon the transducer. Using computational micromechanics Weiland and Leo investigate the effect that ion-ion interaction, pendant chain stiffness, and cluster surface energy have on the charge redistribution within the cluster. Their findings support the macroscopic predictions of the previous models, ${ }^{14,26,28,29,33,34}$ providing justification for the transport concepts used in these larger-scale models.

The present work builds upon many of these existing models, while also trying to address many of the experimental phenomena observed in recent studies. Akle et al. ${ }^{18}$ found that the capacitive properties of an IPT greatly influence the electromechanical response of that transducer. Using a series of ionomer materials with varying degrees of ion concentration, Akle et al. were able to directly relate the electromechanical response of the IPT with its electrical properties. Farinholt and Leo ${ }^{15,35}$ also found this behavior as they related variations in the polymer actuation and sensing response to changes in the transducer's electrical impedance. These changes in the impedance were obtained by controlled variations in the counterion and diluent species within a Nafion ${ }^{\mathrm{TM}}$-based transducer. To better understand the results of these experimental studies, an analytical model has been developed to predict the electrical impedance response of an ionic polymer transducer. This model incorporates the ion diffusion properties, overall transducer permittivity, and environmental parameters to predict surface current of the ionic polymer when excited by an applied voltage.

\section{DEVELOPMENT OF THE CONTINUITY EQUATION}

The underlying theory used to develop this impedance model comes from the well established understanding of ion diffusion through ion-exchange membranes for applications where the membrane separates solutions of different ion concentrations. Lakshminarayanaiah ${ }^{36}$ models the ion flux $\hat{J}$ of an ion species $i$ with a four term expansion of the NernstPlank equation,

$$
\begin{aligned}
\hat{J}_{i}= & -d_{i} \nabla \hat{C}_{i}-d_{i} z_{i} \frac{F \hat{C}_{i}}{R T} \nabla \hat{\phi}_{i} \\
& -\frac{d_{i} \hat{C}_{i}}{R T}\left[M_{i}\left(\frac{V_{i}}{M_{i}}-\frac{V_{w}}{M_{w}}\right)\right] \nabla \hat{p}+\hat{C}_{i} \hat{v},
\end{aligned}
$$

where $d_{i}$ is the ion diffusion coefficient, $\hat{C}_{i}$ is the ion concentration, $z_{i}$ is the ion charge, $\hat{\phi}_{i}$ is the internal electric field, $M_{i}$ and $M_{w}$ are the ion and diluent mass, $V_{i}$ and $V_{w}$ are the ion and diluent volume fraction, $\hat{p}_{i}$ is the external pressure, $\hat{v}$ is the velocity of free diluent, $F$ is Faraday's constant, $R$ is the universal gas constant, $T$ is the ambient temperature, and $\nabla$ is the spatial gradient operator. This expression accounts for the ion flux induced by (1) ion diffusion, (2) an internal potential gradient, (3) an external pressure gradient, and (4) motion of free fluid through the membrane. In the transducer application ion flux can be simplified from the general form shown in Eq. (1). For this study the ionomer is assumed to have the properties of Nafion ${ }^{\mathrm{TM}}-117$, an anionic polymer that is neutralized via positively charged ions. Since the anions are covalently bound to the perfluronated backbone, the only mobile species is the cation species, allowing us to drop the subscript $i$ notation.

The transducer is also designed to operate in either free air or a homogeneous fluid and is plated with a conductive surface electrode. Thus, the transducer is not subject to an external pressure gradient through the ionomer's thickness, while it is also assumed that the motion of free diluent is negligible with respect to the ion flux. In the typical actuation response of the IPT the electric field is applied in the through thickness direction of the ionomer. It is assumed that conduction occurs in the $\hat{x}$ direction. In this manner, the ion flux expression used to represent the ionic polymer transducer has the form

$$
\hat{J}_{i}=-d\left[\frac{\partial \hat{C}^{+}}{\partial \hat{x}}+\frac{F \hat{C}^{+}}{R T} \frac{\partial \hat{\phi}}{\partial \hat{x}}\right] .
$$

From this expression it is seen that the ion flux depends upon the spatial variation of cations as well as the interaction between the cation distribution and the spatial variation of internal potential. With regard to the analysis it is best to rephrase the flux expression in terms of the potential function $\hat{\phi}$. This transformation is accomplished by imposing the definition of charge density $\hat{\rho}=F\left(\hat{C}^{+}-\hat{C}^{-}\right)$, as well as the general field relationships $E=D / \kappa, E=-\nabla \hat{\phi}, \nabla D=\hat{\rho}$, and $\hat{\rho}=\kappa \nabla E$. With these relationships it is possible to express the cation concentration in terms of electric potential $\hat{C}^{+}=-\kappa / F \nabla^{2} \hat{\phi}$ $+C^{-}$. Applying this definition it is possible to express the ion 
flux exclusively in terms of the electric potential

$$
\hat{J}=\frac{d \kappa}{F}\left(\frac{\partial^{3} \hat{\phi}}{\partial \hat{x}^{3}}+\frac{F}{R T}\left[\frac{\partial^{2} \hat{\phi}}{\partial \hat{x}^{2}} \frac{\partial \hat{\phi}}{\partial \hat{x}}\right]-\frac{F^{2} C^{-}}{\kappa R T} \frac{\partial \hat{\phi}}{\partial \hat{x}}\right) .
$$

This expression provides a specific representation of the ion flux within the ionic polymer transducer and is a function of the internal potential. To fully understand the polymer's response it is necessary to relate this ion flux with the rate of ion motion within ionomer membrane. To incorporate the rate of ion motion within this model, it is necessary to impose a continuity condition which relates the rate of cation motion with the spatial gradient of flux

$$
\frac{\partial \hat{C}^{+}}{\partial t}=-\frac{\partial \hat{J}}{\partial \hat{x}} .
$$

The charge density definition and field relationships can again be used to transform Eq. (4) into an expression that is only a function of the internal potential. In this manner, the continuity relationship can be written as

$\frac{\partial}{\partial \hat{t}}\left[\frac{\partial^{2} \hat{\phi}}{\partial \hat{x}^{2}}\right]-d \frac{\partial^{4} \hat{\phi}}{\partial \hat{x}^{4}}-\frac{d F}{R T}\left[\frac{\partial^{3} \hat{\phi}}{\partial \hat{x}^{3}} \frac{\partial \hat{\phi}}{\partial \hat{x}}+\frac{\partial^{2} \hat{\phi}}{\partial \hat{x}^{2}} \frac{\partial^{2} \hat{\phi}}{\partial \hat{x}^{2}}\right]+d \gamma^{2} \frac{\partial^{2} \hat{\phi}}{\partial \hat{x}^{2}}=0$,

where $\gamma^{2}=F^{2} C^{-} / \kappa R T$. Equation (5) provides the governing equation for the electric potential response within the ionic polymer transducer. For this expression to properly model the actuation or impedance response of the transducer, an external voltage must be applied at the outer surfaces $(\hat{x}$ $=0, \hat{x}=L)$ of the IPT. The applied potential is represented by the boundary conditions

$$
\left.\hat{\phi}(\hat{x}, \hat{t})\right|_{\hat{x}=0}=\hat{\Phi}_{1}(\hat{t}) \quad \text { and }\left.\quad \hat{\phi}(\hat{x}, \hat{t})\right|_{\hat{x}=L}=\hat{\Phi}_{2}(\hat{t}),
$$

where $\hat{\Phi}_{1}(\hat{t})$ and $\hat{\Phi}_{2}(\hat{t})$ correspond to the applied potential at $\hat{x}=0$ and $\hat{x}=L$, respectively. In addition to the potential boundary conditions, the polymer is treated as a closed system such that the ion flux is constrained be zero at the outer boundaries of the polymer

$$
\left.\frac{d \kappa}{F}\left(\frac{\partial^{3} \hat{\phi}}{\partial \hat{x}^{3}}+\frac{F}{R T}\left[\frac{\partial^{2} \hat{\phi}}{\partial \hat{x}^{2}} \frac{\partial \hat{\phi}}{\partial \hat{x}}\right]-\frac{F^{2} C^{-}}{\kappa R T} \frac{\partial \hat{\phi}}{\partial \hat{x}}\right)\right|_{\hat{x}=0, \hat{x}=L}=0 .
$$

In the polymer's initial state it is assumed that the cation distribution is at equilibrium such that the charge density $\hat{\rho}$ at time $t=0$ is defined as zero. When expressed in terms of the internal potential this produces the initial condition

$$
-\left.\kappa \frac{\partial^{2} \hat{\phi}(x, t)}{\partial \hat{x}^{2}}\right|_{\hat{t}=0}=0 .
$$

\section{A. Nondimensional form}

To broaden the generality of this model, the continuity relationship is transformed into nondimensional form, producing a system that can be scaled according to geometric and material parameters. To accomplish the nondimensionalization the length scale is normalized by the polymer thick- ness $L$. Similarly, the time constant $\lambda$ is defined to be the characteristic time $d \gamma^{2}$, where $\gamma^{2}$ is defined as the characteristic length $F^{2} C^{-} / \kappa R T$. The potential is normalized by the maximum applied potential $\Phi_{m}=\left|\hat{\Phi}_{2}-\hat{\Phi}_{1}\right|$. Thus, the dimensionless variables $t, x$, and $\phi$ are defined as

$$
x=\frac{\hat{x}}{L}, \quad t=\lambda \hat{t}, \quad \phi=\frac{\hat{\phi}}{\Phi_{m}} .
$$

Applying these variable transformations to Eq. (5) it is possible to develop a dimensionless continuity expression that is subject to nondimensional boundary and initial conditions. This yields a system of the form

$$
\frac{\partial \phi^{\prime \prime}}{\partial t}-\epsilon \phi^{i v}-\epsilon N_{2}\left[\phi^{\prime \prime \prime} \phi^{\prime}+\left(\phi^{\prime \prime}\right)^{2}\right]+\phi^{\prime \prime}=0,
$$

where the $\phi^{\prime}$ notation is used to denote the spatial derivative $\partial \phi / \partial x$, and $\epsilon$ is a dimensionless parameter defined as $1 / L^{2} \gamma^{2}$. Applying the transformation to the boundary conditions, we find that Eq. (10) is subject to the conditions

$$
\begin{aligned}
& x=0 \rightarrow \quad \phi=\Phi_{1} \quad \text { and } \quad N_{1}\left(\epsilon \phi^{\prime \prime \prime}+\epsilon N_{2}\left[\phi^{\prime \prime} \phi^{\prime}\right]-\phi^{\prime}\right)=0, \\
& x=1 \rightarrow \phi=\Phi_{2} \quad \text { and } \quad N_{1}\left(\epsilon \phi^{\prime \prime \prime}+\epsilon N_{2}\left[\phi^{\prime \prime} \phi^{\prime}\right]-\phi^{\prime}\right)=0,
\end{aligned}
$$

where the terms $N_{1}, N_{2}, \Phi_{1}$, and $\Phi_{2}$ are defined as

$$
\begin{aligned}
& N_{1}=\frac{d \kappa \gamma^{2} \Phi_{m}}{F L}, \quad \text { and } \quad N_{2}=\frac{F \Phi_{m}}{R T}, \\
& \Phi_{1}=\frac{\hat{\Phi}_{1}(t)}{\Phi_{m}}, \quad \text { and } \quad \Phi_{2}=\frac{\hat{\Phi}_{2}(t)}{\Phi_{m}} .
\end{aligned}
$$

The system represents the continuity relationship and provides a governing differential equation which describes the spatial and temporal evolution of the internal potential. For the present analysis the goal is to describe the ionic polymer transducer's current response as a function of an external potential. Specifically, this relationship is developed to describe the frequency domain representation of the transducer's electrical impedance. Standard frequency domain analysis assumes that the system is both linear and has reached steady-state. To better understand this steady-state response, we begin by considering the transducer's response to a step voltage excitation.

\section{FORCED RESPONSE-STEP EXCITATION}

In our analysis of the IPT response to a step change in voltage the goal is to study the accumulation of charge at steady-state, while also examining the saturation limits that can be expected at higher excitation levels. In this manner we continue our analysis by considering the linearized form of Eq. (10), yielding the expression

$$
\frac{\partial \phi^{\prime \prime}}{\partial t}-\epsilon \phi^{i v}+\phi^{\prime \prime}=0
$$

While focusing on the steady-state response, the time varying component $\partial \phi^{\prime \prime} / \partial t$ is assumed to be zero, leaving the fourth order differential equation 


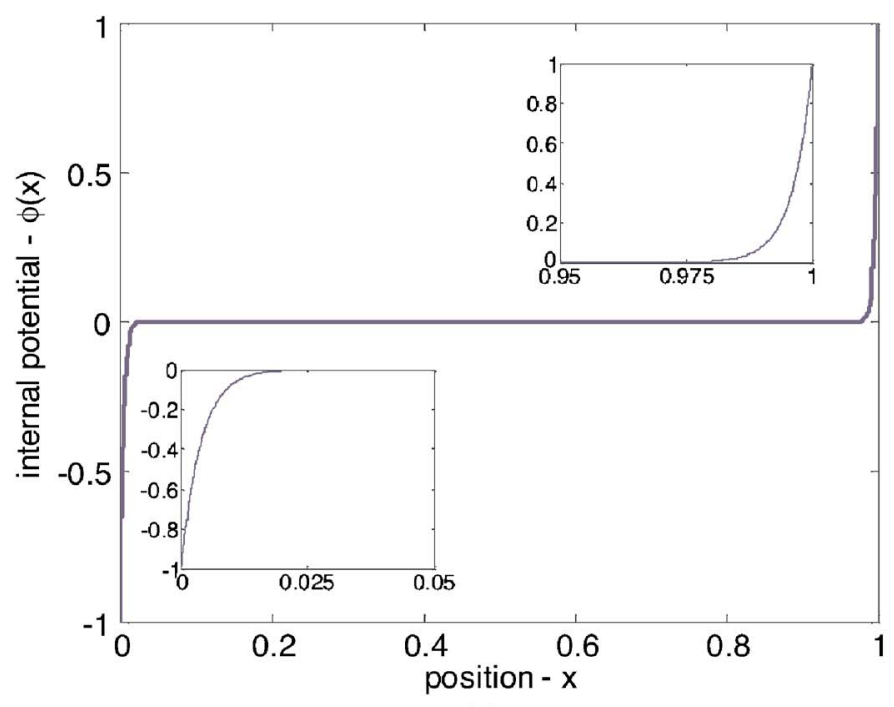

(a)

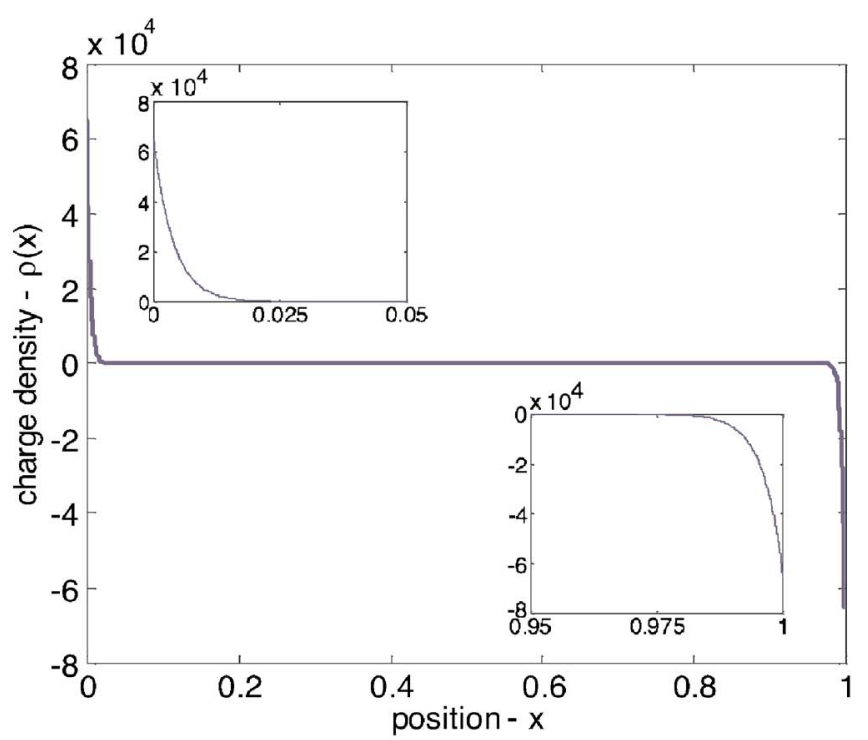

(b)

FIG. 1. (Color online) The steady-state response of the IPT. This figure illustrates the (a) internal potential and (b) charge density through the material thickness.

$$
-\epsilon \phi^{i v}+\phi^{\prime \prime}=0
$$

Since the system is linear, separation of variables is assumed to hold. We define the potential function to be of the form $\phi(x, t)=\varphi(x) \eta(t)$. Applying this definition to Eq. (14) and dividing through by the term $-\epsilon$, we get the expression

$$
\left[\varphi^{i v}(x)-\frac{1}{\epsilon} \varphi^{\prime \prime}(x)\right] \eta(t)=0 .
$$

This analysis will consider the steady-state response to an applied step voltage. In the long term this will represent a constant temporal component. This constant is assigned a value of 1 , allowing us to simply consider the linear differential equation

$$
\frac{\partial^{2}}{\partial x^{2}}\left[\phi^{\prime \prime}(x)-\frac{1}{\epsilon} \phi(x)\right]=0 .
$$

The general solution to this expression has the form

$$
\phi_{\mathrm{ss}}(x)=a_{1} \epsilon \sinh \frac{x}{\sqrt{\epsilon}}+a_{2} \epsilon \cosh \frac{x}{\sqrt{\epsilon}}+a_{3} x+a_{4} .
$$

To continue this linear analysis, we recognize that the solution presented in Eq. (17) is subject to the linear boundary conditions prescribing the applied potential and the ion flux at the membrane boundaries. Since we are considering the steady-state response of the polymer, the boundary conditions will have the form

$$
\begin{aligned}
& x=0 \rightarrow \quad \phi=\Phi_{1} \quad \text { and } \quad N_{1}\left(\epsilon \phi^{\prime \prime \prime}-\phi^{\prime}\right)=0, \\
& x=1 \rightarrow \quad \phi=\Phi_{2} \quad \text { and } \quad N_{1}\left(\epsilon \phi^{\prime \prime \prime}-\phi^{\prime}\right)=0 .
\end{aligned}
$$

Applying these boundary conditions to the general steadystate solution in Eq. (17) it is possible to solve for the constant coefficients $a_{1}-a_{4}$, yielding a solution for the potential of the form

$$
\phi_{\mathrm{ss}}(x)=\frac{1}{2}\left[\left(\Phi_{2}+\Phi_{1}\right)-\frac{\Delta \Phi}{\sinh \frac{1}{2 \sqrt{\epsilon}}} \sinh \frac{1-2 x}{2 \sqrt{\epsilon}}\right],
$$

where $\Delta \Phi$ is defined as $\Phi_{2}-\Phi_{1}$. With this solution it is possible to impose the field relationships to produce an expression which describes the charge density $\rho_{\mathrm{ss}}(x)$. In nondimensional form this relationship is governed by the equation $\rho_{\mathrm{ss}}(x)=-d^{2} \phi_{\mathrm{ss}}(x) / d x^{2}$. Imposing this relationship yields the steady-state solution $\rho_{\mathrm{ss}}(x)$,

$$
\rho_{\mathrm{ss}}=\frac{\Delta \Phi}{2 \epsilon \sinh \frac{1}{2 \sqrt{\epsilon}}} \sinh \frac{1-2 x}{2 \sqrt{\epsilon}} .
$$

These steady-state profiles are shown in detail in Fig. 1. From this figure it is apparent that the internal potential and charge density profiles are dominated by the $\sinh [(1$ $-2 x) / 2 \sqrt{\epsilon}]$ term in Eqs. (20) and (21). These figures also illustrate the boundary layer effect that is predicted to develop within the IPT under a constant external potential. The charge density result is consistent with predictions of Nemat-Nasser, ${ }^{26}$ while Fig. 1(a) extends Nemat-Nasser's results to provide a corresponding prediction of the internal potential as well.

In addition to a qualitative understanding of the IPT response, we extend our analysis to focus on the theoretical potential limit that would correspond to a full depletion of cations near the anode. Following the analysis used to generate Fig. 1, we focus on the cation depletion expected to develop near $x=1$. In this region of the polymer it is evident that the charge density experiences a rapid loss in the number of cations within the boundary layer at $x=1$. As more cations migrate out of this region, the charge density is expected to approach a saturation limit imposed by the anionic nature of the base ionomer itself. The chemical composition of the ionic polymer restricts the minimal charge density to a level corresponding to $\hat{\rho}=-F C^{-}$. In the nondimensional coordinate this saturation limit corresponds to $\rho=-L^{2} F C^{-} / \kappa \Phi_{m}$. There- 
fore, if we apply this saturation constraint to the steady state charge density it is possible to identify a critical potential which will correspond to a full depletion of the cations near the anode. Thus, we begin by considering the case where

$$
\frac{\Delta \Phi}{2 \epsilon \sinh \frac{1}{2 \sqrt{\epsilon}}} \sinh \frac{-1}{2 \sqrt{\epsilon}}=-\frac{L^{2} F C^{-}}{\kappa \Phi_{m}} .
$$

This yields the case where

$$
\frac{-\Delta \Phi}{2 \epsilon}=-\frac{L^{2} F C^{-}}{\kappa \Phi_{m}} .
$$

Imposing the definition for $\Delta \Phi$ in terms of $\hat{\Phi}_{1}, \hat{\Phi}_{2}$, and $\Phi_{m}$, we get an expression in terms of the physical potentials $\hat{\Phi}_{1}$ and $\hat{\Phi}_{2}$,

$$
\frac{\left(\hat{\Phi}_{2}-\hat{\Phi}_{1}\right)}{2 \epsilon \Phi_{m}}=\frac{L^{2} F C^{-}}{\kappa \Phi_{m}} .
$$

We define this critical potential difference as $\Delta \hat{\Phi}_{\text {crit }}=\hat{\Phi}_{2}$ $-\hat{\Phi}_{1}$. Solving for this value we find

$$
\begin{aligned}
& \Delta \hat{\Phi}_{\text {crit }}=\frac{2 \epsilon L^{2} F C^{-}}{\kappa}=\frac{2 L^{2} F C^{-}}{\kappa L^{2} \gamma^{2}}=\frac{2 F C^{-}}{\kappa \frac{F^{2} C^{-}}{\kappa R T}}, \\
& \Delta \hat{\Phi}_{\text {crit }}=\frac{2 R T}{F} .
\end{aligned}
$$

One of the most interesting features of this critical potential is that it relies solely on the ambient temperature $T$. For experiments conducted in the laboratory, this temperature corresponds to $300 \mathrm{~K}$, producing a critical transition potential of

$$
\Delta \hat{\Phi}_{\text {crit }}=51.8 \mathrm{mV}
$$

This critical potential assumes that the charge density has reached a steady-state profile following the application of a step voltage across the material thickness. Such a response offers insight into the boundary layer development near each of the electrodes at $x=0$ and $x=1$ as shown in Fig. 1. Next we want to consider the effects of frequency on the charge density response within the IPT.

\section{FORCED RESPONSE-HARMONIC EXCITATION}

The next step in this development is to consider the IPT steady-state response to a harmonic excitation at the outer boundaries. A steady-state analysis is conducted because the impedance model is intended to predict the frequency response of the ionic polymer's electrical response. Thus, by considering the long term performance we assume the forced response to be the sum of forced and resonant components

$$
\phi(x, t)=F \psi(x) \sin \Omega t+F \delta(x) \cos \Omega t+u(x, t),
$$

where $F$ is defined as $\Delta \Phi / 2$ and $\Omega$ corresponds to the excitation frequency. The functions $\psi(x)$ and $\delta(x)$ are defined as forced modal components. At this point we return our focus to the nondimensional continuity relationship in Eq. (10). One of the underlying assumptions in frequency domain analysis is that the input-output relationship is linearly related. Therefore, the analysis focuses on a linearized version of Eq. (10),

$$
\dot{\phi}^{\prime \prime}-\epsilon \phi^{i v}+\phi^{\prime \prime}=0 .
$$

The assumed solution for the internal potential in Eq. (27) is now applied to the linear partial differential equation. If we define the resonant component to be zero, this yields an expression that can be grouped in terms of $\sin \Omega t$ and $\cos \Omega t$,

$$
\begin{aligned}
& F\left\{\left[-\delta^{\prime \prime}(x) \Omega-\epsilon \psi^{i v}(x)+\psi^{\prime \prime}(x)\right] \sin \Omega t\right. \\
& \left.\quad+\left[\psi^{\prime \prime}(x) \Omega-\epsilon \delta^{i v}(x)+\delta^{\prime \prime}(x)\right] \cos \Omega t\right\}=0 .
\end{aligned}
$$

To satisfy this expression we formulate a set of coupled ordinary differential equations (ODEs) in terms of $\psi$ and $\delta$,

$$
\begin{aligned}
& -\epsilon \psi^{i v}(x)+\psi^{\prime \prime}(x)=\Omega \delta^{\prime}(x), \\
& -\epsilon \delta^{i v}(x)+\delta^{\prime}(x)=-\Omega \psi^{\prime \prime}(x) .
\end{aligned}
$$

The assumed solution for $\phi(x, t)$ in Eq. (27) can also be applied to the boundary conditions to yield a system of eight boundary conditions that can be applied to the system of ODEs in Eq. (30). By applying the boundary conditions and evaluating the system of differential equations, it is possible to solve for the modal functions $\psi(x)$ and $\delta(x)$. This analysis is presented in detail by Farinholt. ${ }^{37}$ These two modal functions constitute the basis for the forced electric potential function. By performing this analysis and substituting the modal functions $\psi(x)$ and $\delta(x)$ into Eq. (27), we develop the following expression for $\phi(x, t)$,

$$
\begin{aligned}
\phi_{f}(x, t)= & \frac{\Delta \Phi}{2 \sqrt{\bar{A}} \Upsilon_{h}}\left\{\operatorname { s i n } \Omega t \left(\sqrt { \overline { A } } \operatorname { s i n h } \frac { \sqrt { \overline { A } } } { 2 } \left[-2 \sinh \frac{\sqrt{A}}{2}(\cosh \sqrt{A} x+\cosh \sqrt{\bar{A}} x)+2 \sinh \sqrt{A} x+\cosh \frac{\sqrt{A}}{2}(\sqrt{A}(\epsilon A-1)(2 x-1)\right.\right.\right. \\
& -\sqrt{A}(\epsilon A-1) \cosh \sqrt{\bar{A}} x)]+\cosh \frac{\sqrt{\bar{A}}}{2}\left[\sinh \frac{\sqrt{A}}{2}(\bar{A}(\epsilon \sqrt{\bar{A}}-1)(2 x-1)-\bar{A}(\epsilon \bar{A}-1) \cosh \sqrt{A} x+2 \sqrt{\bar{A}} \sinh \sqrt{\bar{A}} x)\right. \\
& \left.\left.+\cosh \frac{\sqrt{A}}{2}(\sqrt{A} \sqrt{\bar{A}}(\epsilon A-1) \sinh \sqrt{\bar{A} x}+\bar{A}(\epsilon \bar{A}-1)(\sqrt{A}(\epsilon A-1)(2 x-1)+\sinh \sqrt{A} x))\right]\right) \\
& +\cos \Omega t\left(\sqrt{\bar{A}} \sinh \frac{\sqrt{\bar{A}}}{2}\left[-2(A \cosh \sqrt{A} x+\bar{A} \cosh \sqrt{\bar{A} x}) \sinh \frac{\sqrt{A}}{2}+\cosh \frac{\sqrt{A}}{2}(2 A \sinh \sqrt{A} x-\bar{A} \sqrt{A}(\epsilon A-1) \cosh \sqrt{\bar{A}} x)\right]\right.
\end{aligned}
$$




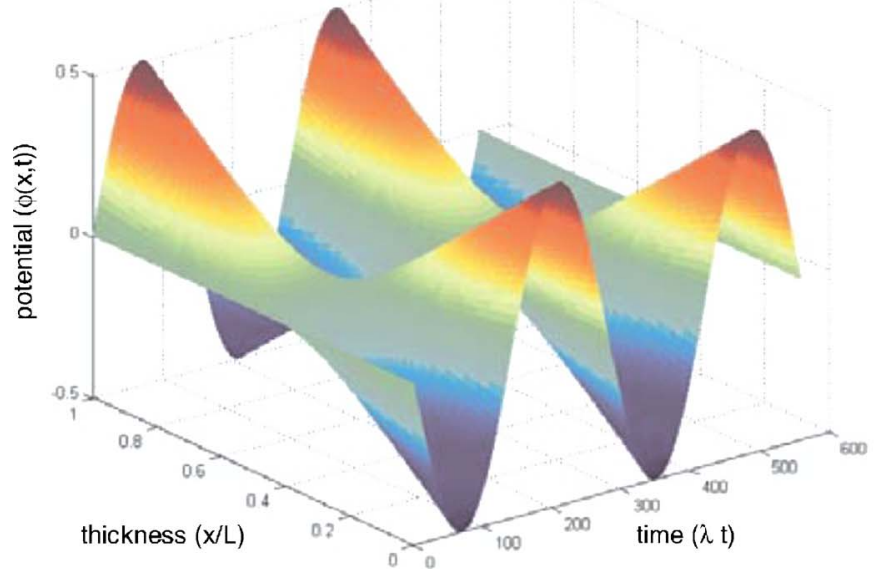

(a)

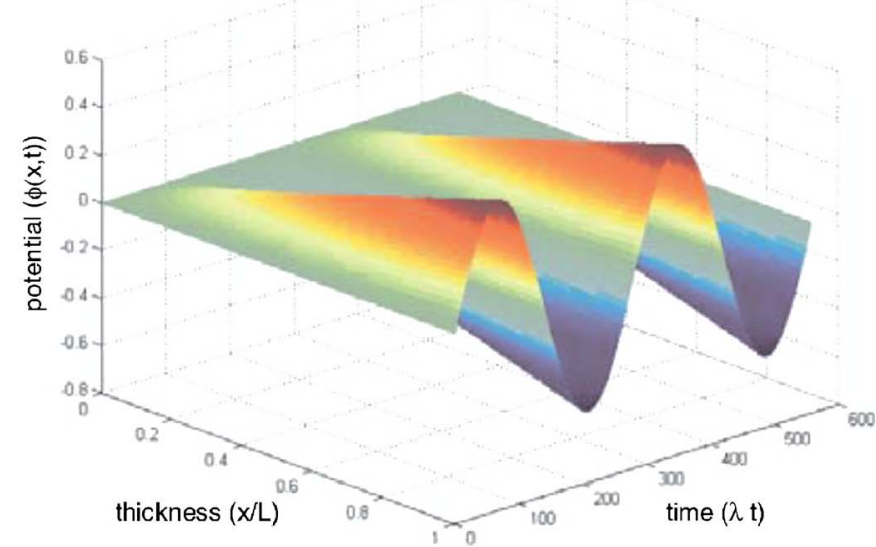

(b)

FIG. 2. (Color online) The electric potential response to an applied harmonic excitation. (a) presents the response to a harmonic excitation at both electrodes, while (b) presents the response to a harmonic excitation at only one electrode. All excitations have unity amplitude and a frequency of $1 \mathrm{~Hz}$.

$$
\begin{aligned}
& +\cosh \frac{\sqrt{\bar{A}}}{2}\left[\sinh \frac{\sqrt{A}}{2}\left(-A \bar{A}(\epsilon \bar{A}-1) \cosh \sqrt{A} x+2 \bar{A}^{3 / 2} \sinh \sqrt{\bar{A}} x\right)\right. \\
& \left.\left.\left.+\cosh \frac{\sqrt{A}}{2}\left(A \bar{A}(\epsilon A-1) \sinh \sqrt{A} x+\sqrt{A} \bar{A}^{3 / 2}(\epsilon A-1) \sinh \sqrt{\bar{A}} x\right)\right]\right)\right\},
\end{aligned}
$$

where the constants $A$ and $\bar{A}$ are defined as $A=(-1 / \epsilon)(1$ $+i \Omega)$ and $\bar{A}=(-1 / \epsilon)(1-i \Omega)$. This internal potential is evaluated through the material thickness and with time, as shown in Fig. 2(a). This plot corresponds to an applied harmonic potential of $\pm(\Delta \Phi / 2) \sin \Omega t$ at each of the electrode surfaces. In this case the potential is of unity magnitude and a frequency $\Omega$ of $1 / \lambda$ or $1 / d \gamma^{2}$. Figure 2(a) shows a nearly linear profile through the material thickness. In addition to the symmetrically applied potential, Fig. 2(b) depicts the internal potential response to a harmonic potential applied at one electrode, while the other is held at ground. This produces a zero internal potential at $x=0$ and a harmonic response at $x=1$ that corresponds to the applied boundary con-

\section{ditifn.}

Using the general electric field relationships it is possible to develop an expression for the forced internal charge den- sity $\rho_{f}(x, t)$, as well as the isothermal transient ionic current (ITIC). An expression for $\rho_{f}(x, t)$ is formulated by developing the nondimensional relationship between charge density and the internal electric potential. Relying on the electric field relationships and the nondimensionalization process of Sec. II A we find that the nondimensional charge density is proportional to the second spatial derivative of the internal potential of the previous section

$$
\rho(x, t)=\frac{L^{2}}{\kappa \Phi_{m}} \hat{\rho}(\hat{x}, \hat{t})=-\frac{\partial^{2} \phi(x, t)}{\partial x^{2}} .
$$

By combining this expression with the results of Eq. (31) it is possible to formulate the internal charge density that is expected to develop for a sinusoidal applied voltage. This external voltage induces charge motion through the polymer thickness, generating an internal potential profile which can be directly related to the forced internal charge density

$$
\begin{aligned}
\rho_{f}(x, t)= & \frac{-\Delta \Phi}{2 \sqrt{\bar{A}} Y_{h}}\left[\operatorname { s i n } \Omega t \left(\sqrt { \overline { A } } \operatorname { s i n h } \frac { \sqrt { \overline { A } } } { 2 } \left[-2 \sinh \frac{\sqrt{A}}{2}(A \cosh \sqrt{A} x+\bar{A} \cosh \sqrt{\bar{A}} x)\right.\right.\right. \\
& \left.+\cosh \frac{\sqrt{A}}{2}(2 A \sinh \sqrt{A} x-\bar{A} \sqrt{A}(\epsilon A-1) \cosh \sqrt{\bar{A} x})\right]+\cosh \frac{\sqrt{\bar{A}}}{2}\left[\sinh \frac{\sqrt{A}}{2}\left(-A \bar{A}(\epsilon \bar{A}-1) \cosh \sqrt{A} x+2 \bar{A}^{3 / 2} \sinh \sqrt{\bar{A}} x\right)\right. \\
& \left.\left.+\cosh \frac{\sqrt{A}}{2}\left(A \bar{A}(\epsilon \bar{A}-1) \sinh \sqrt{A} x+\bar{A}^{3 / 2} \sqrt{A}(\epsilon A-1) \sinh \sqrt{\bar{A} x}\right)\right]\right)
\end{aligned}
$$




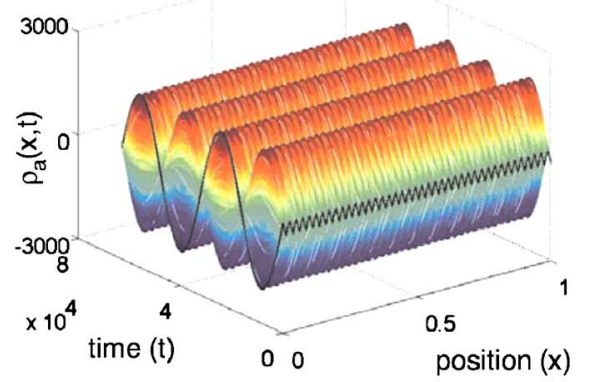

(a)

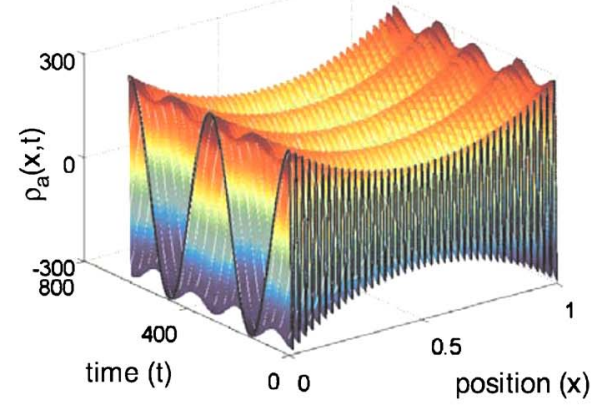

(b)

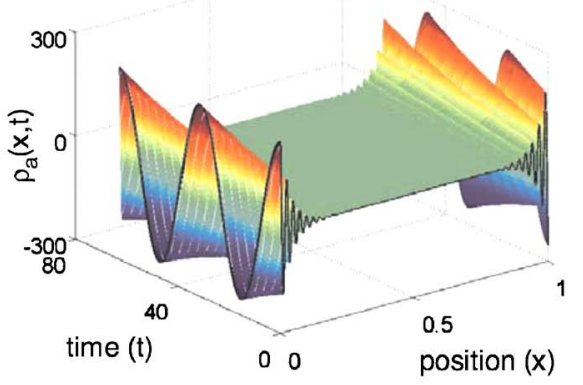

(c)

FIG. 3. (Color online) The charge density response to an applied harmonic excitation. Three separate excitation frequencies are considered: (a) corresponds to $\Omega=0.002$, (b) corresponds to $\Omega=0.02$, while (c) corresponds to $\Omega=0.2$, where $\Omega$ corresponds to the frequency normalized by the characteristic time $\lambda$. All excitations are 0 at $x=0$ and harmonic with amplitude 1 at $x=1$.

$$
\begin{aligned}
& +i \cos \Omega t\left(\operatorname { s i n h } \frac { \sqrt { A } } { 2 } \left[-2 \sqrt{\bar{A}} \sinh \frac{\sqrt{\bar{A}}}{2}(-A \cosh \sqrt{A} x+\bar{A} \cosh \sqrt{\bar{A}} x)\right.\right. \\
& +\cosh \frac{\sqrt{\bar{A}}}{2}\left(2 \bar{A}^{3 / 2} \sinh \sqrt{\bar{A}} x+A \bar{A}(\epsilon A-1) \cosh \sqrt{A} x\right)+\cosh \frac{\sqrt{A}}{2}\left(\sqrt{\bar{A}} \sinh \frac{\sqrt{\bar{A}}}{2}(\bar{A} \sqrt{A}(\epsilon A+1) \cosh \sqrt{\bar{A}} x-2 A \sinh \sqrt{A} x)\right. \\
& \left.\left.\left.+\cosh \frac{\sqrt{\bar{A}}}{2}\left(-A \bar{A}(\epsilon \bar{A}-1) \sinh \sqrt{A} x+\bar{A}^{3 / 2} \sqrt{A}(\epsilon A-1) \sinh \sqrt{\bar{A} x}\right)\right]\right)\right]
\end{aligned}
$$

This expression describes the ion distribution throughout the IPT with respect to space, time, and the frequency of excitation. Figure 3 presents the charge density response that is predicted to develop for three different driving frequencies, ranging from $\Omega=0.0002$ to $\Omega=0.2$. As before $\Omega$ corresponds to the nondimensional frequency that results from normalizing the applied frequency $\omega$ in hertz $(\mathrm{Hz})$ by the characteristic time $\lambda$. At low frequencies the temporal response is in-phase with the applied potential as shown in Fig. 3(a). As this excitation frequency increases the charge density gradually becomes out-of-phase with the input signal, illustrated in Figs. 3(b) and 3(c). Considering the physical performance of the polymer itself, this behavior is expected in the time response. Based on experimental results ${ }^{15}$ the ionic polymer exhibits capacitive properties at low frequencies and resistive properties at higher frequencies, making it natural to expect a shift in phase at higher excitation frequencies. Since the charge density is proportional to the surface charge it is expected that $\rho_{f}(x, t)$ will be in-phase with the applied potential at low frequencies to emulate a capacitive response. Similarly we expect the more resistive, high frequency response to be $90^{\circ}$ out-of-phase with the applied potential, as seen in Fig. 3(c).

In addition to the temporal response of the IPT, the spatial response also exhibits a harmonic profile through the material thickness. At low frequencies this spatial profile has a nearly uniform peak amplitude throughout the polymer. As this excitation frequency increases the magnitude begins to decay near the center of the polymer. This behavior becomes more pronounced as the excitation frequency increases fur- ther and is apparent in Fig. 3(c). Figure 4 considers the spatial response and its characteristic wavelength as they vary exclusively with $x$ and the driving frequency $\Omega$. The spatial profiles of Fig. 4(b) correspond to the time $t$ in which $\rho(x, t)$ is maximized at $x=0$. Six separate excitation frequencies are considered in this plot, ranging from $\Omega=0.0001$ to $\Omega=0.1$. An autospectrum of this data quantifies the characteristic wavelength for each driving frequency, indicating that it is nearly proportional to $2 \pi \sqrt{\epsilon}$ at low excitation frequencies. As the applied frequency increases beyond $\Omega=0.1$, a noticeable shift in the autospectrums becomes evident. Figure 5(a) presents this shift in wavelength, illustrating that the characteristic wavelength increases with applied frequency $\Omega$. Further analysis indicates that this wavelength scales with $2 \pi \sqrt{\epsilon /(1+\Omega)}$, the characteristic length scale in both $\psi(x)$ and $\delta(x)$ of the spatial analysis.

The spatial response of Fig. 5(b) indicates a significant spatial decay in the charge density through the polymer thickness at higher excitation frequencies. Such behavior becomes intuitive as we consider the fundamental nature of the ion-transport membrane. Within the ionomer, ion motion is limited by the material's inherent time constant, which in turn is governed by the diffusion coefficient of the membrane. At low excitation frequencies cations have a significant amount of time to redistribute throughout the transducer's thickness. As this excitation frequency increases cations have less time to migrate through the polymer thickness, and as a result changes in charge density are limited to regions directly adjacent to the electrodes. This localization effect emphasizes the boundary layer region near the polymer sur- 


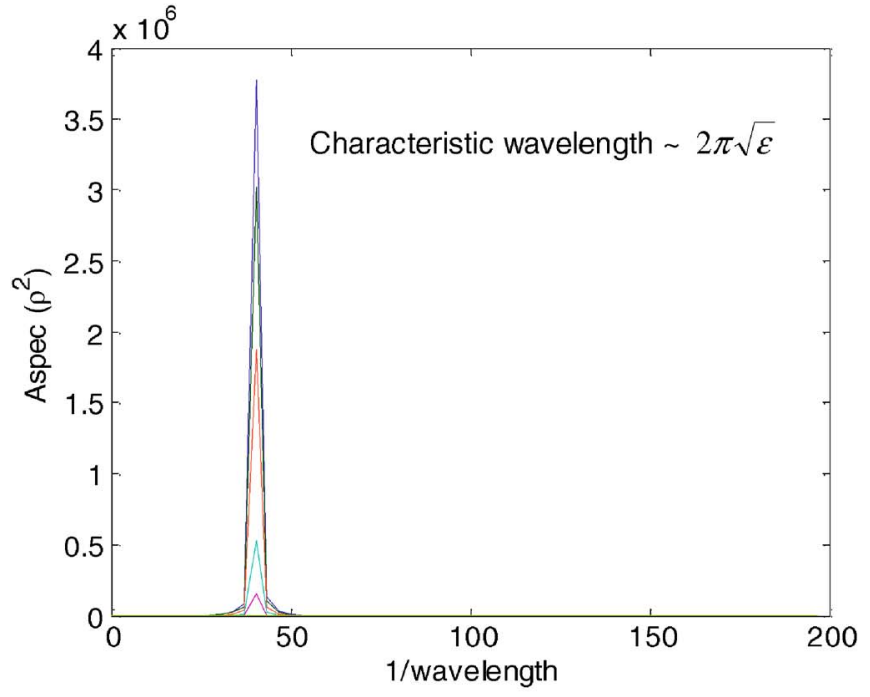

(a)

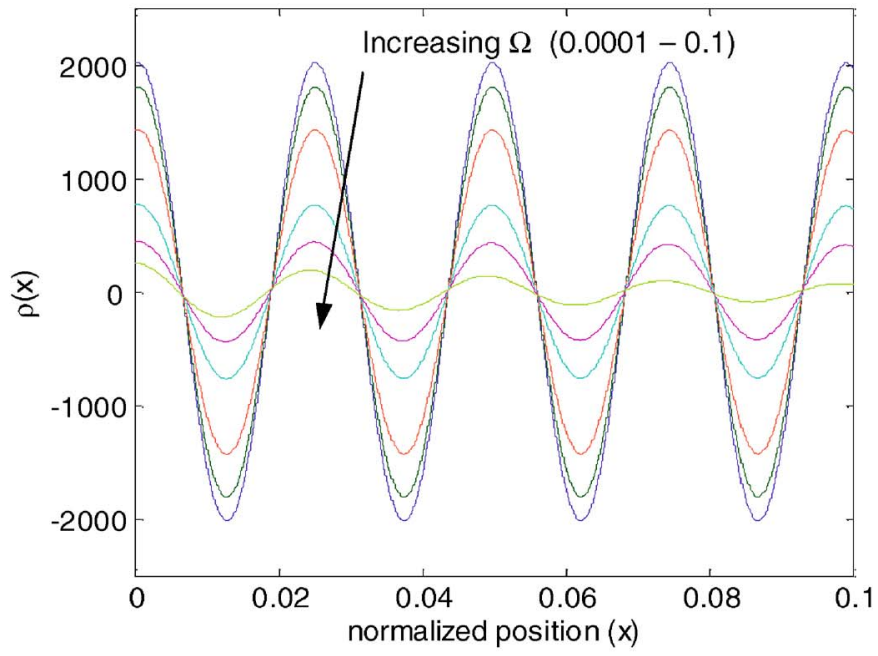

(b)

FIG. 4. (Color online) Wavelength analysis of the charge density's spatial response to low frequency harmonic excitation $(0.0001 \leq \Omega \leq 0.1)$. An autospectrum of the data shows that the characteristic wavelength is $\approx 2 \pi \sqrt{\epsilon}$. Plot (b) presents the spatial distribution of charge density near the boundary at $x=0$ for each of the lower excitation frequencies.

faces at $x=0$ and $x=1$. At low frequencies we notice that the boundary layer is defined by the small scale parameter $\epsilon$, just as it had been in the case of the step excitation discussed previously. As the excitation frequency increases further the boundary layer shifts closer toward the electrode surface, becoming smaller as a function of the driving frequency $\Omega$ through the relationship of $\epsilon /(1+\Omega)$.

\section{B. Saturation limit as a function of frequency and potential}

From the induced charge density we extend our analysis to examine the effects of harmonic excitation on the saturation phenomena that develops at higher excitation levels. In the previous case we considered the saturation effect purely as a function of a step change in the applied voltage. In the

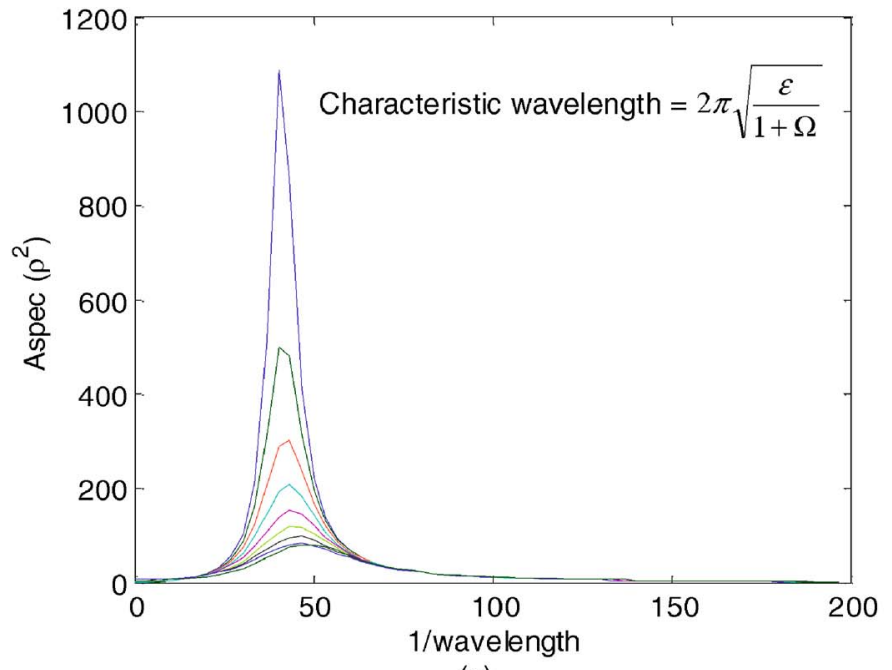

(a) case of a harmonic driving signal we integrate the effects of excitation frequency into our analysis of the saturation region as well. As in our analysis of the step excitation we define saturation to be the level at which the charge density near the anode reaches $\hat{\rho}(\hat{x}, \hat{t})=-F C^{-}$. For a simple step change in the applied voltage, this saturation region was shown to form solely as a function of the applied voltage. In the case of a harmonic excitation frequency is expected to play a key role in determining the saturation level near the anode. Since cation motion is limited by the diffusion coefficient of the polymer, the excitation frequency will determine the duration of time that cations have to accumulate near the cathode. Thus, at lower frequencies cations have longer to migrate through the polymer, resulting in a lower threshold voltage necessary to induce saturation at the IPT

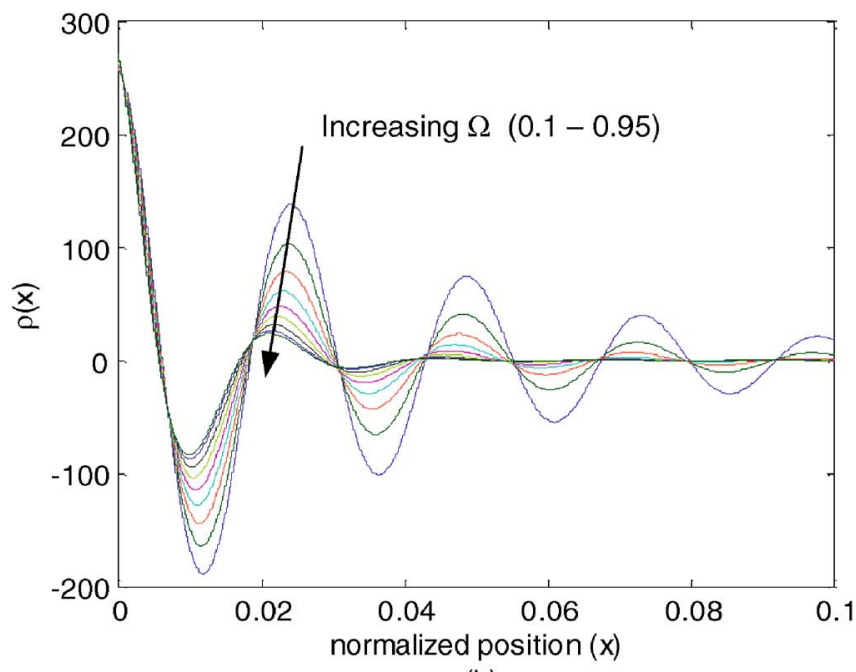

(b)

FIG. 5. (Color online) Wavelength analysis of the charge density's spatial response to higher frequency harmonic excitations $(0.1 \leq \Omega \leq 0.95)$. An autospectrum of the data shows that the characteristic wavelength is equal to $2 \pi \sqrt{\epsilon /(1+\Omega)}$. Plot (b) presents the spatial distribution of charge density near the boundary at $x=0$ for each of the higher excitation frequencies. 


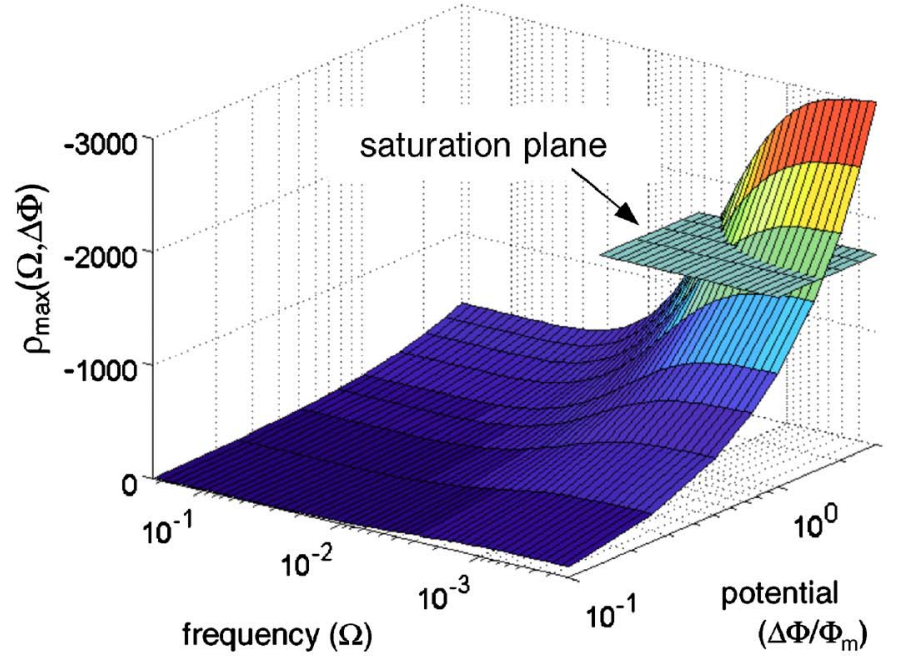

(a)

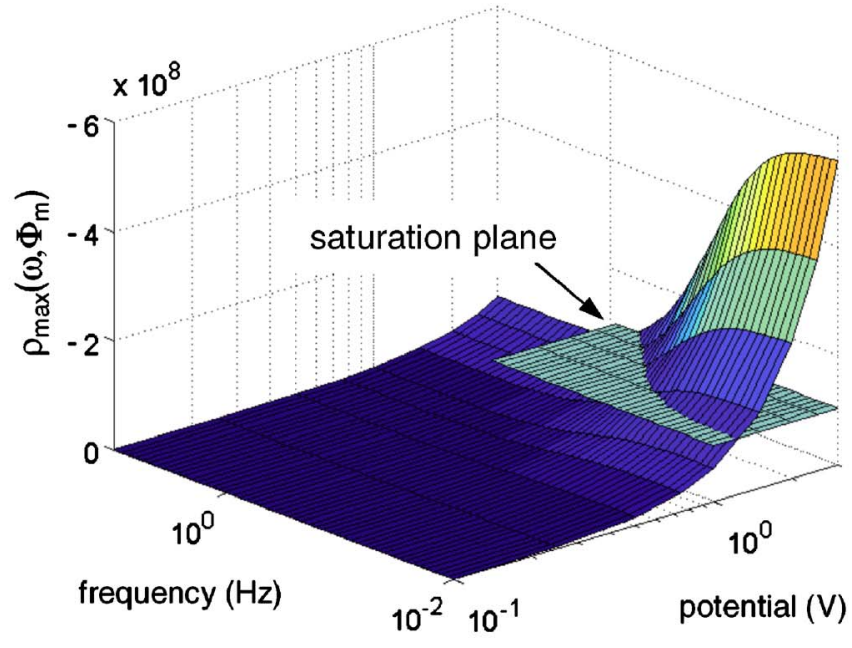

(b)

FIG. 6. (Color online) Peak charge density as a function of applied voltage and frequency. This figure includes both (a) nondimensional and (b) dimensional predictions of the saturation region. Dimensional simulations correspond to a Nafion ${ }^{\mathrm{TM}}-117$ based transducer in $\mathrm{Li}^{+}$form.

anode. Conversely, we expect higher frequencies to require larger applied voltages in order to achieve the same saturation effect.

To analyze the saturation phenomena we begin by considering the peak charge density as a function of the applied potential and driving frequency. The results of this analysis are presented for the nondimensional system in Fig. 6(a), and for the dimensional system in Fig. 6(b). (Note the negative axes.) Superimposed upon each of these figures is a saturation plane that corresponds to the critical charge density corresponding to $\rho_{\text {sat }}(x, t)=-L^{2} F C^{-} / \kappa \Phi_{m}$ in the nondimensional case and to $\hat{\rho}_{\text {sat }}(\hat{x}, \hat{t})=-F C^{-}$in the dimensional case. As the saturation plane intersects the peak charge density, a curve develops which corresponds to the critical frequencypotential relationship that induces saturation at the anode. To formulate this expression we begin with the forced charge density of Eq. (33),

$$
\rho_{f}(x, t)=\frac{-\Delta \Phi}{2 \sqrt{\bar{A}} Y_{h}}[\tilde{\psi}(x) \sin \Omega t+\tilde{\delta}(x) \cos \Omega t],
$$

where $\widetilde{\psi}(x)$ and $\tilde{\delta}(x)$ represent the spatial coefficients of the in-phase and out-of-phase terms of $\rho_{f}(x, t)$. Equating this with the saturation term $\rho_{\text {sat }}(x, t)$ yields an expression in terms of $\Delta \Phi$ and $\Phi_{m}$,

$$
\frac{-\Delta \Phi}{2 \sqrt{\bar{A}} \Upsilon_{h}}[\tilde{\psi}(x) \sin \Omega t+\tilde{\delta}(x) \cos \Omega t]=\frac{-L^{2} F C^{-}}{\kappa \Phi_{m}} .
$$

Imposing the definition $\Delta \Phi=\left(\hat{\Phi}_{2}-\hat{\Phi}_{1}\right) / \Phi_{m}$, we can solve for the critical potential difference $\Delta \hat{\Phi}=\hat{\Phi}_{2}-\hat{\Phi}_{1}$ as

$$
\Delta \hat{\Phi}=\frac{2 \sqrt{\bar{A}} Y_{h} L^{2} F C^{-}}{\kappa[\tilde{\psi}(x) \sin \Omega t+\tilde{\delta}(x) \cos \Omega t]} .
$$

This expression describes the critical potential difference as a function of space $(x)$, time $(t)$, and the driving frequency $(\Omega)$. In this analysis we are most concerned with the potential corresponding to the peak charge density induced at the anode. Therefore, we express the critical potential as a function of applied frequency by evaluating Eq. (36) at $x$ $=x_{\text {anode }}$ and at the peak time $t=t_{p}$,

$$
\Delta \hat{\Phi}=\left.\frac{2 L^{2} F C^{-} \sqrt{\bar{A}} Y_{h}}{\kappa[\tilde{\psi}(x) \sin \Omega t+\tilde{\delta}(x) \cos \Omega t]}\right|_{\substack{t=t_{p} \\ x=x_{\text {anode }}}} .
$$

The peak time specified in this expression is a function of frequency and will therefore change with $\Omega$. This phase shift is evident in Fig. 3 and ranges between $0^{\circ}$ and $-90^{\circ}$ depending on the excitation frequency $\Omega$. This phase relationship becomes very important in the next analysis which models the surface charge and current that develops at the IPT electrodes.

\section{Surface charge and current}

In addition to the saturation analysis, we can use the internal charge density to develop models of the surface charge and current that would be measured at the outer surfaces of the polymer. Greeuw and Hoenders ${ }^{38}$ present an analytical approach to describe the transient ionic current that develops in a dielectric medium subject to an external voltage. Using this work as a basis, we define the general form of the isothermal transient ionic charge $Q_{\mathrm{it}}(t)$ to be the spatial integral of $\hat{x}$ times the charge density $\hat{\rho}(\hat{x}, \hat{t})$ through the material thickness

$$
\hat{Q}_{\mathrm{it}}(\hat{t})=\frac{1}{L} \int_{0}^{L} \hat{x} \hat{\rho}(\hat{x}, \hat{t}) d \hat{x} .
$$

This definition corresponds to the physical system and therefore has not been nondimensionalized. Using the coordinate transformations outlined in Table I and the nondimensional form of the charge density in Eq. (32), we express the transient charge as 
TABLE I. Relationship between physical and the nondimensional terms used in this analysis.

\begin{tabular}{|c|c|c|c|c|c|}
\hline Quantity & Physical variable & Dimensionless expression & Quantity & Physical variable & Dimensionless expression \\
\hline Position & $\hat{x}$ & $x=\frac{\hat{x}}{L}$ & Charge density & $\hat{\rho}(\hat{x}, \hat{t})$ & $\rho(x, t)=\frac{L^{2}}{\kappa \Phi_{m}} \hat{\rho}(\hat{x}, \hat{t})$ \\
\hline Time & $\hat{t}$ & $t=\lambda \hat{t}$ & Surface charge & $\hat{Q}_{\mathrm{it}}(\hat{t})$ & $Q_{\mathrm{it}}(t)=\frac{L}{\kappa \Phi_{m}} \hat{Q}_{\mathrm{it}}(\hat{t})$ \\
\hline Potential & $\hat{\phi}(\hat{x}, \hat{t})$ & $\phi(x, t)=\frac{\phi(\hat{x}, \hat{t})}{\Phi_{m}}$ & Surface current & $\hat{I}_{\mathrm{it}}(\hat{t})$ & $I_{\mathrm{it}}(t)=\frac{L}{\kappa \lambda \Phi_{m}} \hat{I}_{\mathrm{it}}(\hat{t})$ \\
\hline
\end{tabular}

$$
\hat{Q}_{\mathrm{it}}(\hat{t})=\frac{1}{L} \int_{0}^{1} L x \frac{\kappa \Phi_{m}}{L^{2}} \rho(x, t) L d x=\frac{\kappa \Phi_{m}}{L} \int_{0}^{1} x \rho(x, t) d x .
$$

From this the relationship between the dimensional and nondimensional forms of the isothermal transient ionic charge can be represented as

$$
Q_{\mathrm{it}}(t)=\frac{L}{\kappa \Phi_{m}} \hat{Q}_{\mathrm{it}}(\hat{t}) .
$$

Thus, we can express the nondimensional isothermal transient ionic charge as a function of either the charge density $\rho(x, t)$ or the internal potential $\phi(x, t)$,

$$
Q_{\mathrm{it}}(t)=\int_{0}^{1} x \rho(x, t) d x=-\int_{0}^{1} x \frac{\partial^{2} \phi(x, t)}{\partial x^{2}} d x
$$

Similarly we can consider the ITIC in its nondimensional form. Since current is defined as the time derivative of charge, the ITIC will have the form

$$
\hat{I}_{\mathrm{it}}(\hat{t})=\frac{d \hat{Q}_{\mathrm{it}}(\hat{t})}{d \hat{t}}
$$

Substituting our definitions for $\hat{Q}$ and $d / d \hat{t}$ into this expression, we find that the current has the form

$$
\hat{I}_{\mathrm{it}}(\hat{t})=\frac{\kappa \lambda \Phi_{m}}{L} \frac{d Q_{\mathrm{it}}(t)}{d t} .
$$

Therefore, we nondimensionalize $\hat{I}_{\mathrm{it}}(t)$ by $L / \kappa \lambda \Phi_{m}$ to give us a unitless form of the ITIC,

$$
\begin{aligned}
I_{\mathrm{it}}(t) & =\frac{d Q_{\mathrm{it}}(t)}{d t}=\frac{d}{d t}\left[\int_{0}^{1} x \rho(x, t) d x\right] \\
& =-\frac{d}{d t}\left[\int_{0}^{1} x \frac{\partial^{2} \phi(x, t)}{\partial x^{2}} d x\right] .
\end{aligned}
$$

The isothermal current defined in this expression represents the surface current as a function of the induced charge density within the IPT. Applying the forced charge density relationship $\rho_{f}(x, t)$ from Eq. (33) we get the isothermal transient ionic current

$$
\begin{aligned}
I_{\mathrm{it}}(t)= & \frac{-\Delta \Phi}{\sqrt{\bar{A}} \Upsilon_{h}}\left(\operatorname { c o s h } \frac { \sqrt { \overline { A } } } { 2 } \sqrt { \overline { A } } \left\{A \cosh \frac{\sqrt{A}}{2}[(\epsilon a-1) \Omega \cos \Omega t\right.\right. \\
& +b \epsilon \Omega \sin \Omega t]-\sqrt{A} \sinh \frac{\sqrt{A}}{2}(2 \epsilon \bar{A} \Omega \cos \Omega t \\
& +i \epsilon \bar{A} \Omega \sin \Omega t)\}-\sin \frac{\sqrt{\bar{A}}}{2}\left\{A \cosh \frac{\sqrt{A}}{2}[\Omega(\epsilon A\right.
\end{aligned}
$$

$$
\left.\left.\begin{array}{l}
-2) \cos \Omega t-i \epsilon A \Omega \sin \Omega t] \\
+4 \sqrt{A} \sinh \frac{\sqrt{A}}{2}(\Omega \cos \Omega t)
\end{array}\right\}\right) .
$$

Physically this surface current represents the measurable current generated by an external potential applied across the ionic polymer's thickness. With this expression it is possible to construct an equivalent admittance model of the IPT by relating the input voltage to the predicted surface current. Consequently, we can invert this admittance model to produce an impedance model of the ionic polymer. Thus, it is possible to sweep through a series of applied frequencies, noting the magnitude and phase of $I_{\mathrm{it}}(t)$ and $\Phi(t)$ to generate an impedance response of the polymer, as shown in Fig. 7(a).

When compared to the measured response of a Nafion ${ }^{\mathrm{TM}}$ sample tested in the laboratory, the general form of the impedance response is very similar, as seen in Fig. 7(b). Each of these responses is similar in form to a conventional $R C$ electrical system. Specifically, the low frequency response of the IPT is indicative of a capacitive component which results in a gradual roll-off in the magnitude response while the current is $60^{\circ}-90^{\circ}$ out-of-phase with the applied voltage. At higher frequencies this response becomes more resistive as the magnitude begins to level off and the current and voltage signals become in-phase with one another. These characteristics have been observed in many experimental studies ${ }^{11,18,37}$ and are evident in the modeling results of this work. The next sections will place more effort on understanding the capacitive and resistive components of this model and how internal parameters such as the overall permittivity $\left(\kappa_{e}\right)$ and diffusion coefficient $(d)$ influence the predicted impedance response.

\section{COMPARISON WITH EQUIVALENT CIRCUIT MODELS OF THE IPT}

In modeling many active materials it is often beneficial to consider an equivalent circuit model that exhibits similar response characteristics. For the ionic polymer transducer several $R C$ models have been proposed to describe the material response. Shahinpoor ${ }^{39}$ presented a simple $R C$ model of the polymer gel assuming a resistive and capacitive component in series. Paquette et al. ${ }^{40}$ presented a more detailed circuit model which treats the electrode regions as a parallel $R C$ circuit and the bulk polymer as a purely resistive element. A simple impedance representation of each of these models takes the form

$$
Z_{T}=\frac{s R C+1}{s C},
$$



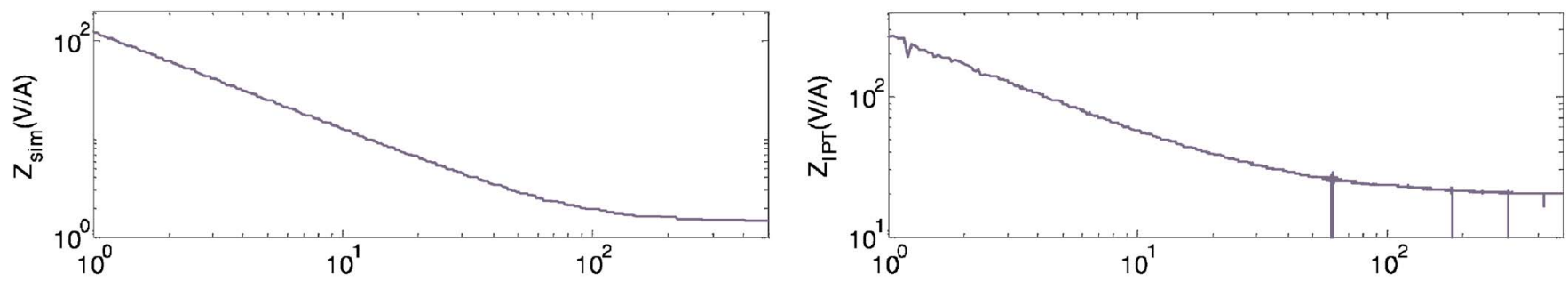

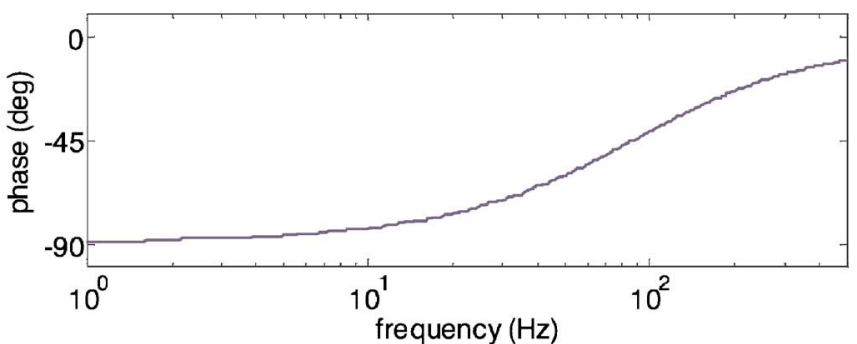

(a)

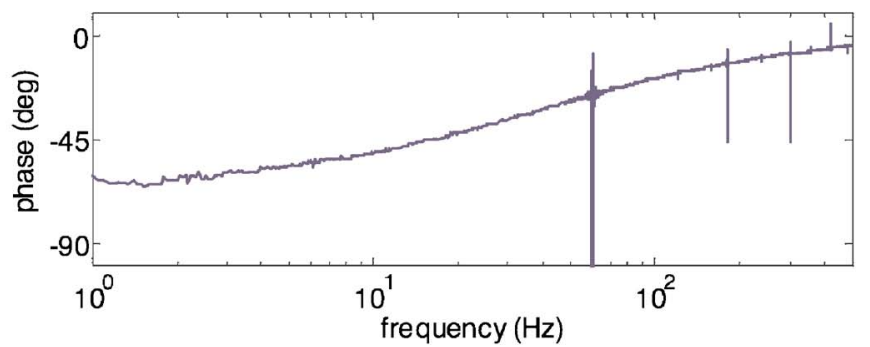

(b)

FIG. 7. (Color online) Frequency response results for the electrical impedance of an ionic polymer transducer. Plot (a) depicts the simulated relationship between the calculated ITIC and the applied voltage while plot (b) presents the measured response for an experimental sample (Nafion ${ }^{\mathrm{TM}}-117$ polymercation: $\mathrm{Li}^{+}$, diluent: $\mathrm{H}_{2} \mathrm{O}$ ).

$$
Z_{T}=\frac{s R_{m} R_{e} C_{e}+2 R_{e}+R_{m}}{s R_{e} C_{e}+1}
$$

where $R$ and $C$ are the effective resistance and capacitance, $R_{e}, C_{e}, R_{m}$, and $C_{m}$ are those for the electrode and membrane regions, and $s$ is the Laplace variable.

For the analytical model developed in this work, the electrical impedance relates the isothermal transient ionic current $I_{\mathrm{it}}(t)$ from Eq. (43) to an applied harmonic voltage. Since the polymer in this model is driven by the sinusoidal excitation $\Phi(t)=\frac{1}{2} \Delta \Phi \sin \Omega t$, it is evident that there are two principal components to the $I_{\mathrm{it}}(t)$ : an in-phase term related to $\sin \Omega t$ and an out-of-phase term corresponding to $\cos \Omega t$. Separating the ITIC in this manner yields the in-phase $I_{\mathrm{ip}}(t)$ component

$$
\begin{aligned}
I_{\mathrm{ip}}(t)= & \frac{-\epsilon \Delta \Phi \Omega}{\sqrt{A} \Upsilon_{h}}\left(\operatorname { c o s h } \frac { \sqrt { \overline { A } } } { 2 } \sqrt { \overline { A } } \left[A b \cosh \frac{\sqrt{A}}{2}\right.\right. \\
& \left.\left.-i \sqrt{A} \sqrt{\bar{A}} \sinh \frac{\sqrt{A}}{2}\right]+i A^{2} \cosh \frac{\sqrt{A}}{2} \sinh \frac{\sqrt{\bar{A}}}{2}\right) \sin \Omega t,
\end{aligned}
$$

as well as the out-of-phase $I_{\mathrm{op}}(t)$ component,

$$
\begin{aligned}
I_{\mathrm{op}}(t)= & \frac{-\Delta \Phi \Omega}{\sqrt{A} Y_{h}}\left(\operatorname { c o s h } \frac { \sqrt { \overline { A } } } { 2 } \sqrt { \overline { A } } \left[A(\epsilon a-1) \cosh \frac{\sqrt{A}}{2}\right.\right. \\
& \left.-\sqrt{A}(\epsilon \bar{A}-2) \sinh \frac{\sqrt{A}}{2}\right]+\sinh \frac{\sqrt{\bar{A}}}{2}[A(\epsilon A \\
& \left.\left.-2) \cosh \frac{\sqrt{A}}{2}-4 \sqrt{A} \sinh \frac{\sqrt{A}}{2}\right]\right) \cos \Omega t .
\end{aligned}
$$

By using the simple $R C$ analogy for the polymer in Eq. (44), we can conceptually associate the in-phase component with the pseudoconductance $\left(R^{-1}\right)$ of the polymer. Similarly, we can correlate the out-of-phase component with the pseu- dosusceptance $(2 \pi f C)$ of the system. The nomenclature of conductance and susceptance are used in this case because the polymer is being driven by the applied voltage $\Phi(t)$, resulting in an induced current $I_{\mathrm{it}}(t)$. The corresponding admittance can be expressed as

$$
Y=\sqrt{G^{2}+B^{2}}=\frac{I}{V}=\frac{I_{\mathrm{ip}}(t)+I_{\mathrm{op}}(t)}{\Phi(t)},
$$

where $G$ is the conductance and $B$ is the susceptance of the polymer. From this expression we see that the conductance and susceptance cannot simply be taken as the ratios $I_{\mathrm{ip}}(t) / \Phi(t)$ or $I_{\mathrm{op}}(t) / \Phi(t)$, respectively. Expanding the admittance from Eq. (48) we see that $G$ and $B$ are related to the in-phase and out-of-phase terms of the ITIC through the expression

$$
G^{2}+B^{2}=\frac{\left[I_{\mathrm{ip}}(t)+I_{\mathrm{op}}(t)\right]^{2}}{\Phi^{2}(t)}=\frac{I_{\mathrm{ip}}^{2}(t)+2 I_{\mathrm{ip}}(t) I_{\mathrm{op}}(t)+I_{\mathrm{op}}^{2}(t)}{\Phi^{2}(t)} .
$$

From this expansion the conductance $G$ is defined as the component of $I_{\mathrm{ip}}^{2}(t)+2 I_{\mathrm{ip}}(t) I_{\mathrm{op}}(t)+I_{\mathrm{op}}^{2}(t)$ that is in-phase with the applied potential, while the susceptance is defined as the out-of-phase component of $I_{\mathrm{ip}}^{2}(t)+2 I_{\mathrm{ip}}(t) I_{\mathrm{op}}(t)+I_{\mathrm{op}}^{2}(t)$. At this point the coupling term $2 I_{\mathrm{ip}}(t) I_{\mathrm{op}}(t)$ becomes important in determining the equivalent resistance and capacitance of the polymer. Since this term is related to $G^{2}+B^{2}$, the actual conductance and susceptance will be related to $\sqrt{2 I_{\mathrm{ip}}(t) I_{\mathrm{op}}(t)}$. Looking closely at this term we find that it cycles between real and imaginary values with time, while also introducing a phase shift of $-45^{\circ}$ into the analysis. The presence of this term greatly complicates the analytical analysis, and therefore we redirect our focus to consider a complex impedance method to describe the equivalent resistance and capacitance of the model. In this manner we define the impedance to be the inverse of the admittance $Z=1 / Y$ in accordance with fundamental electric theory, giving us the relationship 

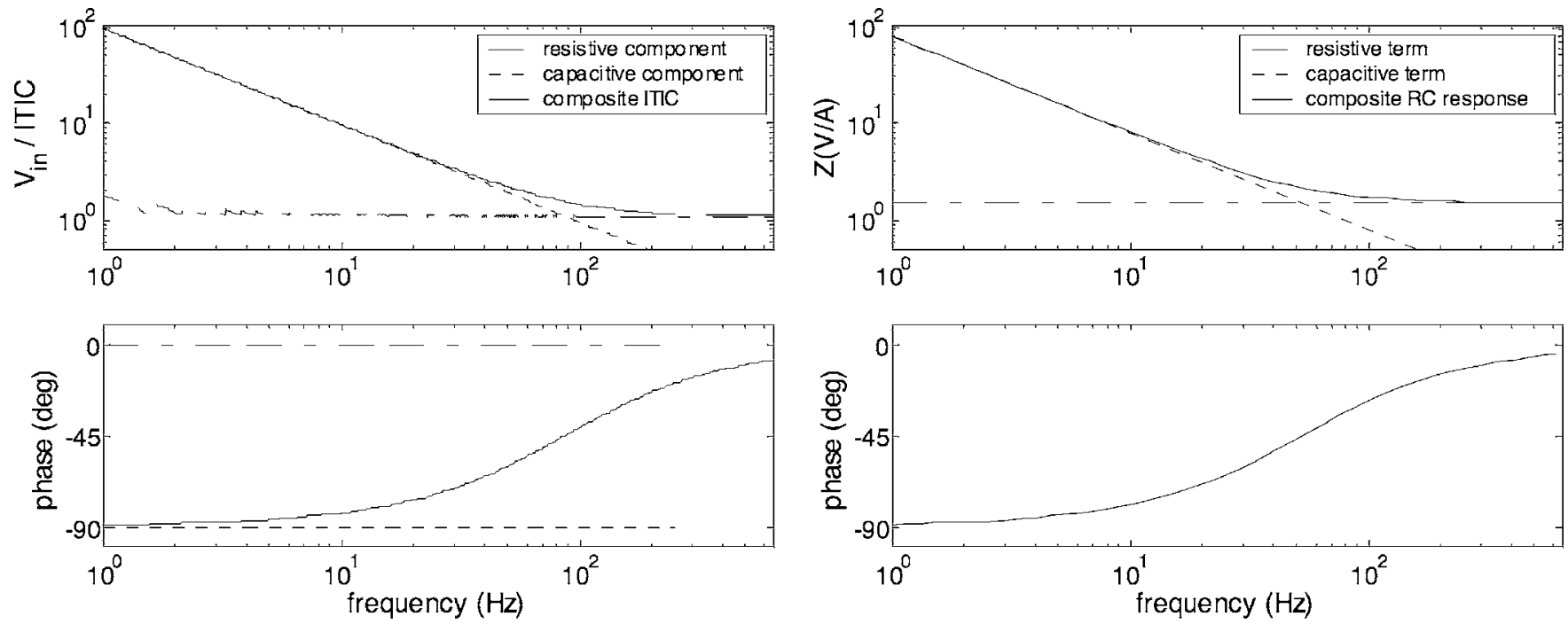

(a)

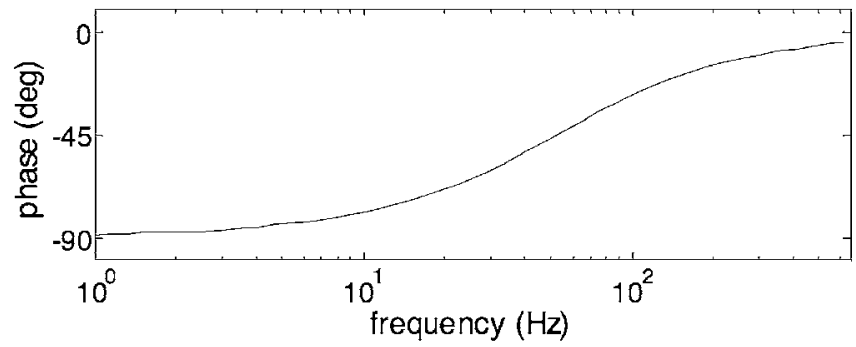

(b)

FIG. 8. Impedance response broken into resistive and capacitive. Results correspond to a dimensional study of (a) the IPT model and (b) the simple $R C$ circuit from Eq. (44).

$$
Z=\frac{V}{I}=\frac{\Phi(t)}{I_{\mathrm{it}}(t)} .
$$

Using a sine-dwell approach, we progress through the impedance analysis extracting magnitude and phase information between $\Phi(t)$ and $I_{\mathrm{it}}(t)$, as functions of the applied frequency $\Omega$. From this magnitude and phase information we construct a complex impedance representation defined as $Z$ $=\bar{Z} e^{i \theta}$, where $\bar{Z}$ is the ratio of peak magnitudes of $\Phi(t)$ and $I_{\mathrm{it}}(t)$, and $\theta$ defines the phase between $\Phi(t)$ and $I_{\mathrm{it}}(t)$. The complex impedance developed using this technique contains the same information used to generate the impedance response of Fig. 7. The benefit of this representation is that we can now employ equivalent circuit models to extract profiles for the equivalent resistance and capacitance. Using the series representation $Z=R+1 / j \omega C$ we extract the equivalent $R$ and $C$ components shown in Fig. 8. The frequency response shown in Fig. 8(a) presents the resistive $\left(Z_{r}\right)$, capacitive $\left(Z_{c}\right)$, and total $(Z)$ impedance curves based upon the series representation of an equivalent $R C$ circuit. From this figure we see that the total impedance tracks the capacitive response at low frequencies, then transitions to a resistive response beyond $\Omega=2$. From the resistive $Z_{r}$ and capacitive $Z_{c}$ impedance profiles, we can extract equivalent $R=Z_{r}$ and $C=-1 / \Omega Z_{c}$ values for the system. Figure 8(a) also illustrates that the equivalent $R$ and $C$ values change very little with respect to driving frequency $\Omega$. The capacitive response is nearly constant, while the resistance does exhibit a slight decay with increased frequency. There also appears to be a slight ripple in the low frequency resistance response, most evident in the region $\Omega \leq 0.5$. This feature diminishes with higher resolution in the time simulations and is therefore attributed to the step size used to evaluate the $\Phi(t)$ and $I_{\mathrm{it}}(t)$ expressions.

We can extend the complex impedance method to consider the physical IPT system by redimensionalizing the $I_{\mathrm{it}}(t)$ and $\Phi(t)$ expressions. In their dimensional form the equivalent resistance is predicted to be $1.2 \Omega$ at high frequencies, with an equivalent capacitance of $2 \mathrm{mF}$. The ionic polymer is modeled as a water based Nafion ${ }^{\mathrm{TM}}-117$ ionomer having $\mathrm{Li}^{+}$ counterions whose physical parameters are found in Table II. The electrical impedance response for this system is shown in Fig. 8(a). The equivalent capacitance $(2 \mathrm{mF})$ and resistance $(1.2 \Omega)$ values overlay on the predicted impedance response, illustrating that the polymer's capacitance dominates the low frequency response, while the resistance dominates the high frequency response. Using a series $R C$ circuit such as the one described in Eq. (44), it is seen that the circuit's electrical impedance [Fig. 8(b)] closely follows the predictions of the proposed model and the simulation results illustrated in Fig. 8(a).

\section{ANALYTICAL STUDY-PARAMETER EFFECTS}

In the previous section our analysis focused on the equivalent electrical properties of the ionic polymer transducer. In this section the focus shifts toward understanding the internal parameters of the ionomer membrane. We now want to consider the influence of material and environmental parameters on the voltage and current relationship within the ionic polymer transducer. We begin this part of the analysis by identifying three primary variables of interest: the diffusion coefficient $d$, the electric permittivity $\kappa$, and the ambient temperature $T$. The first two variables where chosen because they govern the polymer's ability to transport ions $(d)$ and its

TABLE II. Parameters used in modeling the Nafion ${ }^{\mathrm{TM}}$-based transducer.

\begin{tabular}{lcccc}
\hline Cation & \% vol gain & $\begin{array}{c}\mathrm{C}^{-} \\
\left(\mathrm{mol} / \mathrm{m}^{3}\right)\end{array}$ & $\begin{array}{c}\text { Diffusion coefficient } \\
\left(\mathrm{m}^{2} / \mathrm{s}\right)\end{array}$ & $\begin{array}{c}\kappa_{e} \\
(\mathrm{mF} / \mathrm{m})\end{array}$ \\
\hline $\mathrm{Li}^{+}$ & 61.7 & 1070 & $1.389 \times 10^{-10}$ & 2.04 \\
$\mathrm{Na}^{+}$ & 44.3 & 1200 & $1.030 \times 10^{-10} \mathrm{~b}$ & 1.88 \\
$\mathrm{~K}^{+}$ & 18.7 & 1460 & $7.30 \times 10^{-11 \mathrm{~b}}$ & 1.28 \\
$\mathrm{Cs}^{+}$ & 13.6 & 1530 & $1.94 \times 10^{-11 \mathrm{~b}}$ & 1.32 \\
\hline \hline
\end{tabular}

Taken from Ref. 48.

${ }^{\mathrm{b}}$ Taken from Ref. 49 

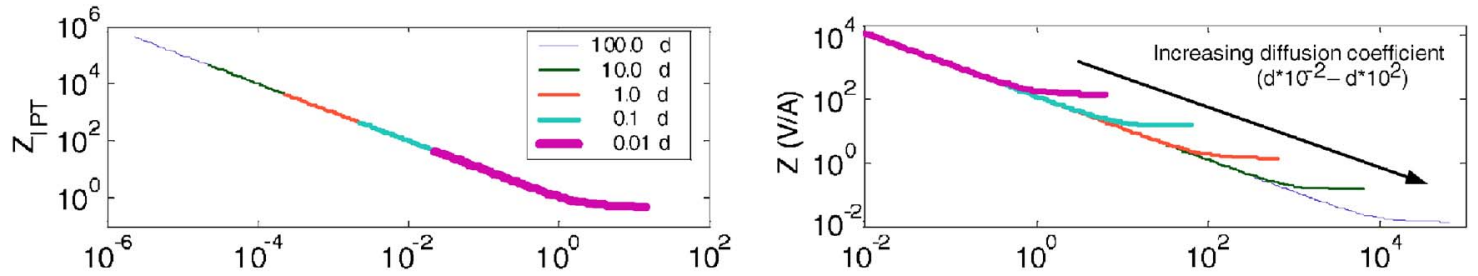

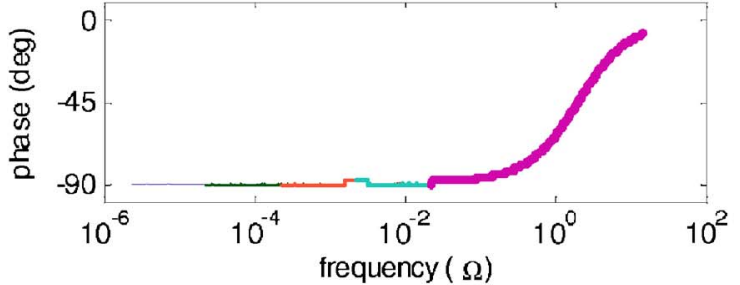

(a)

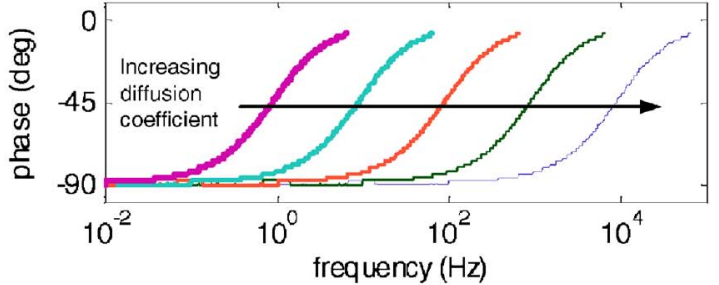

(b)

FIG. 9. (Color online) Impedance response as a function of the diffusion coefficient $d$. The effect of the diffusion coefficient is not very evident in the nondimensional case (a). However, the dimensional case (b) indicates shifts in both magnitude and break frequency as a function of the $d$.

ability to store charge $(\kappa)$. The temperature variable is chosen because it relates to the ionomer's interaction with its environment.

\section{A. Diffusion coefficient-d}

The first variable considered in this parametric study is the membrane's diffusion coefficient $d$ with results shown in Fig. 9. This term defines the counterions ability to move within, and through, the transport membrane and variations in this parameter are expected to influence the resistive nature of the IPT. In this analysis the reference value for the diffusion coefficient is taken to be the self-diffusion rate of a hydrated Nafion ${ }^{\mathrm{TM}}-117$ membrane in $\mathrm{Li}^{+}$form operating at $25^{\circ} \mathrm{C}$. The nominal value for this self-diffusion coefficient is $d=1.389 \times 10^{-10} \mathrm{~m}^{2} / \mathrm{s}$. Figure 9 presents the IPT impedance response for five variations in the diffusion coefficient, spanning from $0.01 \mathrm{~d}$ to $100 \mathrm{~d}$. In the nondimensional case of Fig. 9(a) there is no measurable difference between the simulated impedance response that is calculated for each of the diffusion coefficients. This invariance to the diffusion coefficient is a result of the normalization process used to nondimensionalize the general continuity expression. By normalizing the temporal coefficient by the system time constant $\lambda=d\left(F^{2} C^{-} / \kappa R T\right)$, we effectively remove the diffusion coefficient from the nondimensional analysis. Therefore, we must consider the dimensional, or physical, representation of the ITIC to examine the effects of $d$ on the transducer's impedance response. From the physical representation shown in Fig. 9(b) it is evident that the resistive component changes dramatically as a function of $d$. As the diffusion coefficient increases, a steady decline in the material's resistive behavior is observed. In this manner the capacitive nature of the polymer dominates over a larger frequency band, driving the cutoff frequency higher as the diffusion coefficient increases.

From a physical standpoint, an increased diffusion coefficient translates into enhanced mobility of the counterion within the ionic polymer membrane. This increased mobility indicates that cations can diffuse through the ionomer at a higher rate. Thus, there is a decrease in opposition to the cation migration through the ionic polymer and therefore a lower resistance at higher diffusion coefficients.

\section{B. Effective permittivity- $\kappa$}

The second parameter considered in this analysis is the effective permittivity $\kappa$ of the transducer. Figure 10(a) presents the impedance response for four scalings of the permittivity, from $1 \kappa$ to $1000 \kappa$, where $\kappa$ is taken to be $2 \mathrm{mF} / \mathrm{m}$. As in the case of the diffusion coefficient, the nondimensionalization process removes any influence that the permittivity has on the normalized response. Therefore, the results presented in Fig. 10(a) only consider the physical, or dimensional, system. From this figure it can be seen that $\kappa$ scales the capacitive component of the impedance response while maintaining a constant high frequency resistance. Since the permittivity of a pure dielectric is directly related to capacitance, it makes sense that $\kappa$ 's predominant effect is on the low frequency capacitance of the ionic polymer. This shift in the material's capacitance causes a significant shift in the time constant of the polymer. The time constant itself, $\lambda$ $=d F^{2} C^{-} / \kappa R T$, is inversely related to the effective permittivity, indicating that higher values of $\kappa$ should result in a decrease in $\lambda$. This trend is evident in Fig. 10(a) as the corner frequency of the polymer decreases steadily with increasing permittivity. In terms of the overall modeling approach, the effective permittivity provides a capacitance related term that functions independently from the diffusion coefficient considered in the previous analysis. While each of these parameters affect the transducer's time constant, they scale the overall shape of the impedance in distinctly different ways. Therefore, the parameters $d$ and $\kappa$ provide the ability to tune the resistive and capacitive characteristics of the impedance response independently, a feature which will be beneficial in predicting the effective properties of experimental measurements.

\section{Temperature-T}

The final parameter considered in this analysis is the ambient temperature $T$. While holding the other coefficients 

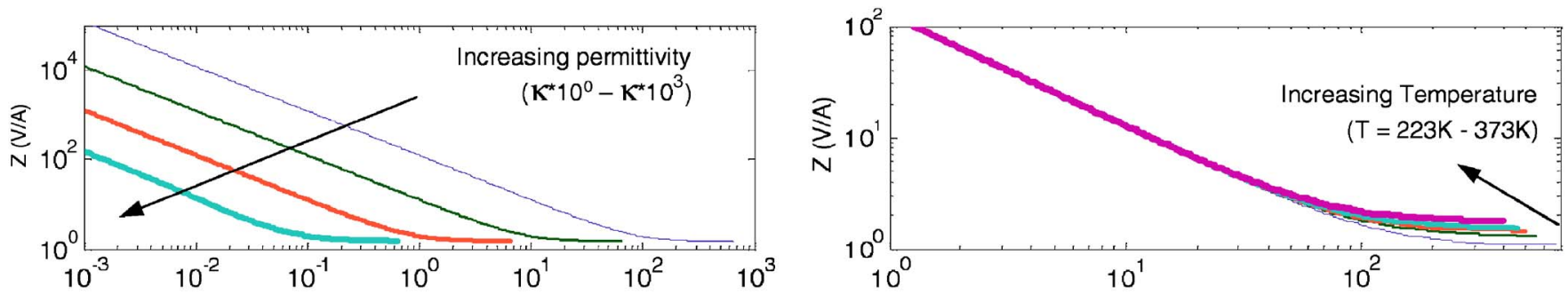

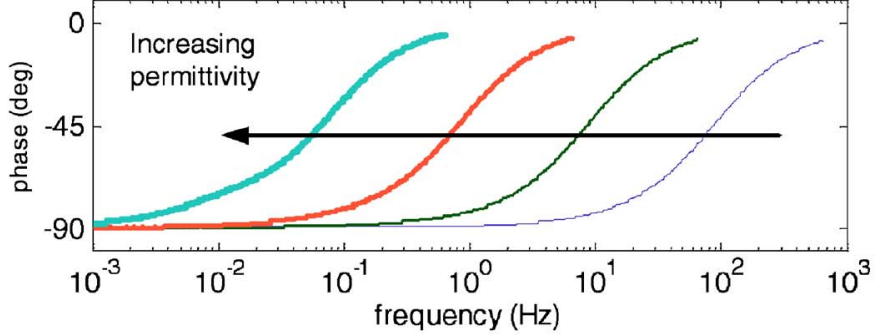

(a)

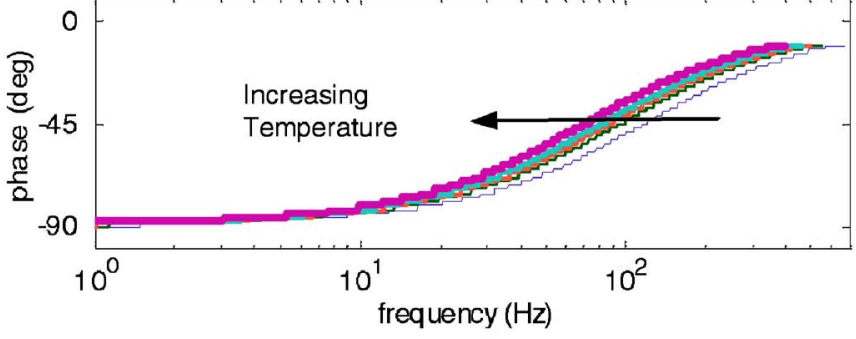

(b)

FIG. 10. (Color online) Impedance response as a function of (a) the electrical permittivity $\kappa$ and (b) the ambient temperature $T$. Results are presented for the dimensional case.

constant, the effects of temperature are very consistent with those of the electric permittivity discussed in the previous case. Figure 10(b) illustrates the impedance response as a function of temperature. Since the scalability of temperature is limited to a relatively small range, we consider temperature variations from 223 to $373 \mathrm{~K}$. We see that the system's time constant $\lambda$ scales inversely with $T$, in much the same manner as it did with $\kappa$. Similarly we note that the polymer becomes more resistive with increasing temperature, and that $\lambda$ subsequently decreases as well. While these results come directly from the model development, we must recognize that the predictions do not follow trends observed in initial experimental results. These experiments indicate that the IPT's high frequency resistance actually decreases with a rise in temperature, contrary to the predictions of the model. To account for this discrepancy we revisit our model, incorporating interactions between the ambient temperature $T$ and the diffusion coefficient $d$. Yeager et al. ${ }^{41,42}$ conducted several experimental studies that looked at the self-diffusion coefficient of $\mathrm{Na}^{+}$and $\mathrm{Cs}^{+}$through Nafion ${ }^{\mathrm{TM}}$ as a function of the ambient temperature. Yeager et al. found that the diffusion coefficient had a strong dependence on temperature for each of the counterions considered. A table of their results is shown in Table III, and those values for the $\mathrm{Na}^{+}$counterion are shown graphically in Fig. 11(a). Using the values of Yeager et al. we now consider the polymer's impedance as a function of temperature while incorporating the temperature variant diffusion coefficient for $\mathrm{Na}^{+}$. The results shown in Fig. 11(b) indicate that temperature changes in the diffusion coefficient actually cause $d$ to dominate the impedance response of the polymer. Figure 11(b) illustrates that the high frequency resistance should decrease with rising temperature due to coinciding increases in the diffusion coefficient. This response in particular will be investigated further in the experimental study of the next section, at which point direct comparisons will be made between analytical predictions and experimental results.

\section{EXPERIMENTAL STUDY}

The culmination of the modeling approach in this study is the development of an expression for the isothermal transient ionic current in response to a harmonic voltage signal applied to the polymer's outer electrode. This model of the surface current provides us with the ability to predict the ionic polymer's electrical impedance as a function of five principal terms: anion concentration $C^{-}$, polymer thickness $L$, ambient temperature $T$, diffusion coefficient $d$, and the

TABLE III. Diffusion coefficients as a function of temperature. Values are from work by Yeager and Steck (Ref. 42).

\begin{tabular}{|c|c|c|c|c|c|}
\hline \multirow[b]{2}{*}{ Ion } & \multirow[b]{2}{*}{ Temp $\left({ }^{\circ} \mathrm{C}\right)$} & \multicolumn{4}{|c|}{ Diffusion coefficients $\left(\mathrm{m}^{2} / \mathrm{s}\right)$} \\
\hline & & I & I & I & II \\
\hline \multirow[t]{3}{*}{$\mathrm{Na}^{+}$} & 0 & $3.07 \times 10^{-11}$ & $2.78 \times 10^{-11}$ & & $3.18 \times 10^{-11}$ \\
\hline & 25 & $9.44 \times 10^{-11}$ & $11.2 \times 10^{-11}$ & & $9.83 \times 10^{-11}$ \\
\hline & 40 & $15.1 \times 10^{-11}$ & $14.9 \times 10^{-11}$ & & $14.8 \times 10^{-11}$ \\
\hline \multirow[t]{3}{*}{$\mathrm{Cs}^{+}$} & 0 & $3.84 \times 10^{-13}$ & $3.80 \times 10^{-13}$ & $4.46 \times 10^{-12}$ & $3.63 \times 10^{-12}$ \\
\hline & 25 & $5.20 \times 10^{-12}$ & $1.70 \times 10^{-11}$ & $2.38 \times 10^{-11}$ & $1.88 \times 10^{-11}$ \\
\hline & 40 & $1.58 \times 10^{-11}$ & $3.37 \times 10^{-11}$ & $3.01 \times 10^{-11}$ & $2.67 \times 10^{-11}$ \\
\hline
\end{tabular}




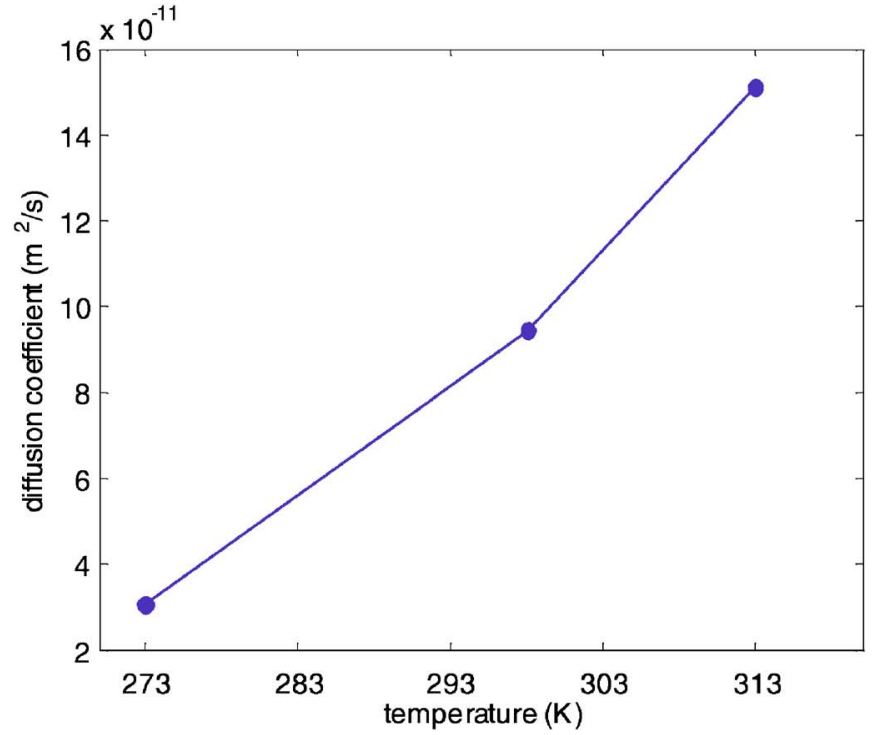

(a)
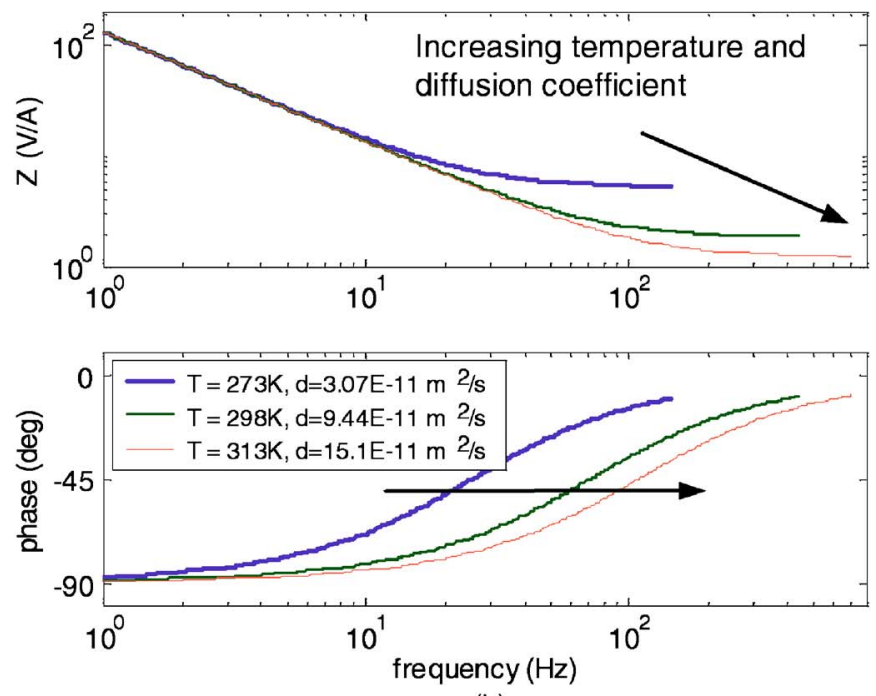

(b)

FIG. 11. (Color online) Impedance response as a function of the external temperature $T$ and a temperature dependent diffusion coefficient $d$. This figure considers the response of a membrane in $\mathrm{Na}^{+}$form. The figure includes (a) diffusion as a function of temperature and (b) the predicted impedance as a function of $T$ and $d$.

transducer's effective permittivity $\kappa$. In this section the focus shifts toward the physical system, looking at the experimental impedance as a function of the ambient temperature, diluent type, and mechanical loading.

\section{A. Impedance as a function of ambient temperature}

Numerous studies of ionic polymers have shown that the transport properties vary as a function of the ambient temperature. Some of the initial work performed in this area was conducted by Yeager et $a l^{41,42}$ who investigated the selfdiffusion properties of Nafion ${ }^{\mathrm{TM}}$ as a function solution temperatures. From their experimental work Yeager et al. found that the ion mobility declines with temperature, resulting in lower diffusion coefficients at lower temperature scales. Paquette and $\mathrm{Kim}^{43-45}$ went on to consider the effect of subzero temperatures on Nafion ${ }^{\mathrm{TM}}$ based ionic polymer-metal composites (IPMC). Focusing on the actuation response, Paquette and Kim found that the IPMC transducer still exhibits an actuation response below the freezing temperature of the diluent (water), however, they found that the blocked force response declines with decreasing temperature. They attributed this feature to the formation of water crystals within the ionomer, a result that offers agreement with findings of Asaka et al. $^{46}$

In this portion of the study we consider the ionic polymer transducer's response to temperatures above and below room temperature. In the parametric study of Sec. VI, we recognize the importance of the relationship between diffusion properties and ambient temperatures in ionomer membranes. Incorporating the diffusion results of Yeager et al. we find that the high frequency resistance is predicted to decrease with increasing temperature in the parametric study of Sec. VI C. Now we consider how these predictions correlate with experimental measurements. Figure 12 presents the experimental setup used in this investigation. A dSPACE 1005 controller with DS2103 digital-to-analog converter and DS2002/2003 MUX analog-to-digital boards was used for signal generation and measurement of the voltage and current responses of the ionic polymer sample. The transducer was driven with a chirp voltage signal of $0.5 \mathrm{~V}, 0-1000 \mathrm{~Hz}$

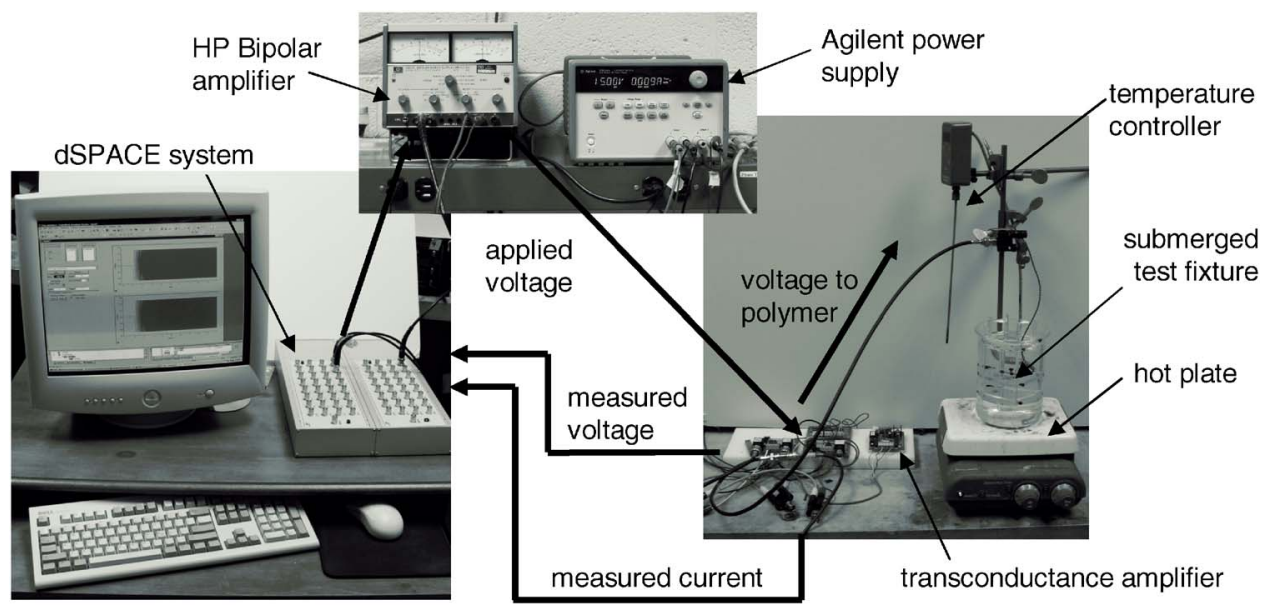

FIG. 12. (Color online) Test fixture used to test the ionic polymer's electrical impedance at various temperatures. 

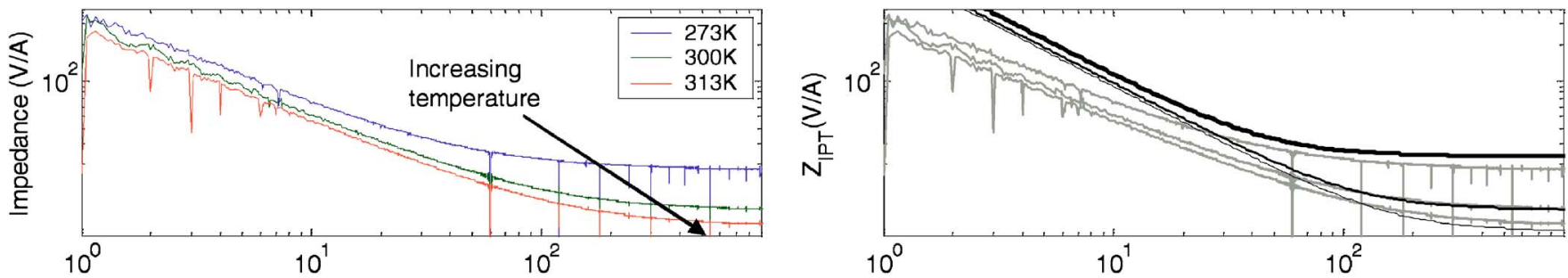

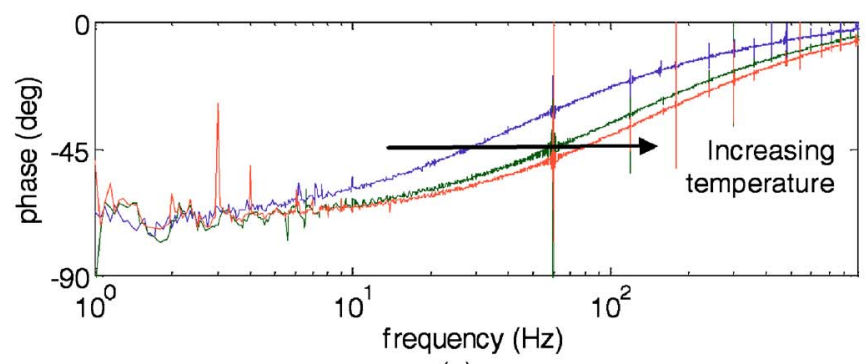

(a)

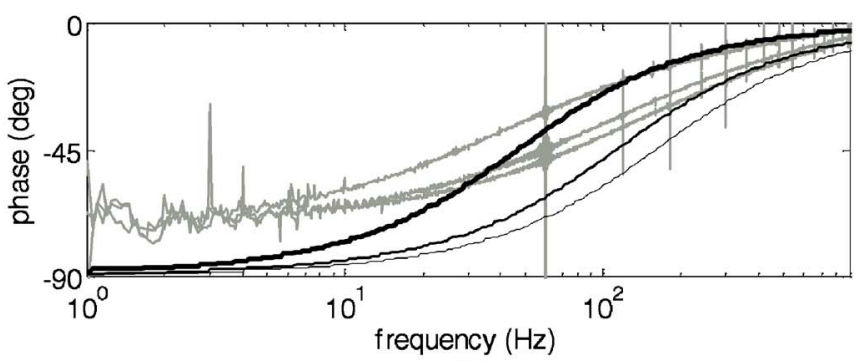

(b)

FIG. 13. (Color online) Impedance response of an IPT in $\mathrm{Li}^{+}$form as a function of temperature, ranging from 274 to $313 \mathrm{~K}$. Plot (a) presents the results measured directly from the experiment while (b) presents the results as normalized by the low frequency component of the sample at $274 \mathrm{~K}$. In the comparison of (b) the gray curves correspond to experimental data and black curves correspond to model predictions.

through an HP 6825A bipolar power supply/amplifier. A transconductance amplifier, ${ }^{47}$ powered by an Agilent E3648A dual output direct current (dc) power supply, was used to condition the voltage signal into the ionic polymer, and to measure the current drawn by the polymer as tests were run. The polymer itself was mounted between fixed electrodes in a fixture that was submerged in de-ionized water. Temperature of the water was controlled using a Corning hot plate and a feedback Corning temperature controller. An ice bath was used for measurements below room temperature. A standard mercury thermometer was used to verify the temperature of the water bath in each case. Three temperature levels were considered in these experiments, corresponding to 0,25 , and $40{ }^{\circ} \mathrm{C}$. These temperature levels were chosen to correspond to the diffusion studies of Yeager et al. summarized in Table III.

In this experiment the IPT was placed in a water bath as the temperature was regulated to the desired level. Once the water bath had reached the target temperature, the system was allowed to equilibrate for $30 \mathrm{~min}$ before testing began. The testing procedure began at $0{ }^{\circ} \mathrm{C}$ and then increased to 25 and $40{ }^{\circ} \mathrm{C}$, subsequently. All tests were run consecutively to minimize sample variation. The results of this analysis are shown in Fig. 13(a). From this set of experiments we see that the ambient temperature has a strong effect on the steadystate resistance of the IPT, showing strong agreement with the predictions of Fig. 11 in Sec. VI C. Looking closely at the low frequency response of Fig. 13(a) we see a slight shift in magnitude of the response. This feature is attributed to an increase in the capacitive term in the impedance, which is directly related to the effective permittivity $\kappa$ of the material. Thus, by looking at the frequency domain response of the electrical impedance we note that the increased temperature must result in a rise in both the diffusion coefficient and the effective permittivity of the membrane. More specifically, we conclude that the increased temperature has a larger effect on $d$ than it does on the permittivity $\kappa$. This observation is based on the behavior of the corner frequency and phase information of the impedance response. From the experimental results of Fig. 13(a) we see that the corner frequency continually increases with rising temperature, as does the phase transition of the impedance. This shift in corner frequency is indicative of an increase in $d$. From the parametric study of Sec. VI, we see that the permittivity has an opposing influence on the corner frequency, as related in the system's time constant $\lambda$,

$$
\lambda=d \gamma^{2}=d \frac{F^{2} C^{-}}{\kappa R T} .
$$

From this expression we see that the material's time constant is proportional to the material's diffusion coefficient and inversely proportional to the effective permittivity and temperature. Therefore, the diffusion coefficient must be the dominant mechanism in order for the corner frequency of the impedance to increase in the steady manner represented in Fig. 13(a).

If we apply this reasoning to the modeling approach, we can generate predictive impedance curves that are superimposed upon the data as shown in Fig. 13(b). In this series of simulations the impedance at room temperature $T=298 \mathrm{~K}$ is used as the base line. The impedance model is initially tuned to the baseline response by fixing the temperature to $T$ $=298 \mathrm{~K}$ and tuning the diffusion coefficient $d$ and permittivity $\kappa$ to match the experimental response. From this initial fit,

TABLE IV. Model parameters used to simulate impedance as a function of pretension.

Pretension Temperature Electric permittivity Diffusion coefficient Gain

\begin{tabular}{rcccc}
\multicolumn{1}{c}{$\mathrm{N})$} & $T(\mathrm{~K})$ & $\kappa(\mathrm{F} / \mathrm{m})$ & $d\left(\mathrm{~m}^{2} / \mathrm{s}\right)$ & \\
\hline 27.92 & 293 & $2.64 \times 10^{-3}$ & $1.82 \times 10^{-10}$ & 0.132 \\
102.32 & 293 & $2.60 \times 10^{-3}$ & $1.43 \times 10^{-10}$ & 0.132 \\
152.12 & 293 & $2.58 \times 10^{-3}$ & $1.17 \times 10^{-10}$ & 0.132 \\
\hline \hline
\end{tabular}




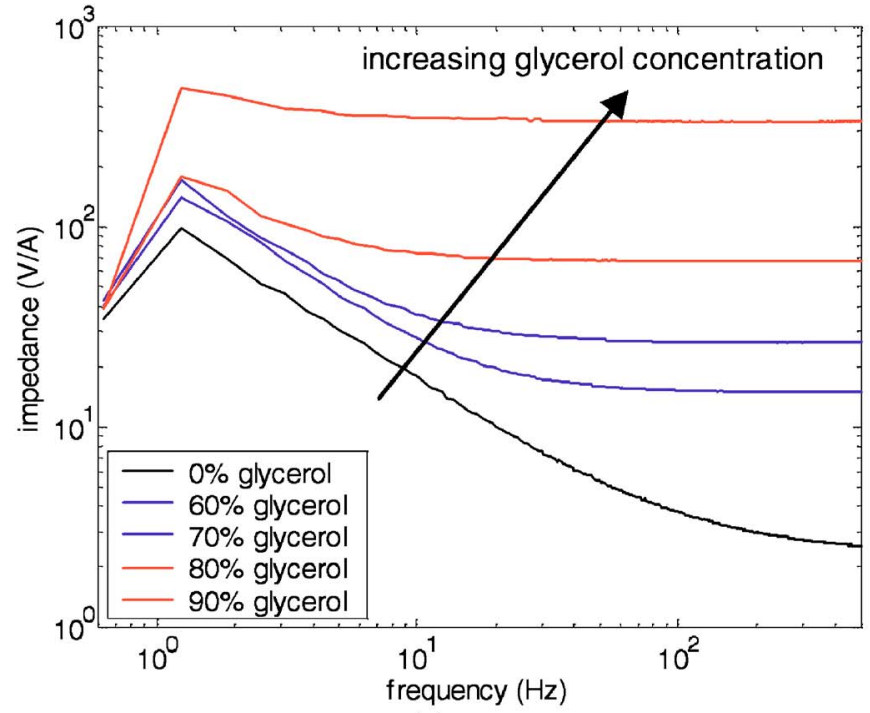

(a)
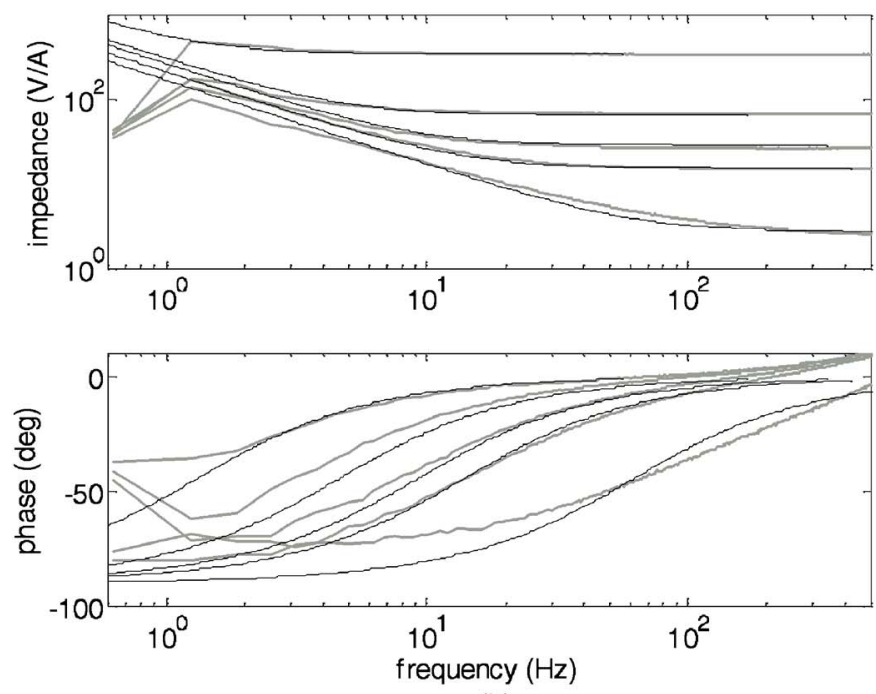

(b)

FIG. 14. (Color online) Impedance response of an IPT as a function of diluent viscosity, ranging from 1.01 to $265.98 \mathrm{cp}$. Plot (a) presents the results measured directly from the experiment while (b) presents a comparison with model predictions. In the comparison of (b) the gray curves correspond to experimental data and black curves correspond to model predictions.

the temperature is defined by the experimental conditions and the diffusion coefficient is scaled following the relationship prescribed by Yeager et al., ${ }^{42}$ and illustrated in Fig. 11(a). Therefore, the free variable in this analysis was the effective permittivity $\kappa$. This parameter was initially fit for the base line response at $T=298 \mathrm{~K}$. For each of the subsequent tests the permittivity was tuned to scale the low frequency impedance. Only slight variations in $\kappa$ (presented in Table IV were used to tune the predicted response. By applying these values to the model we obtain the results in Fig. 13(b) which are superimposed on the experimental results from Fig. 13(a). Comparing the experimental and analytical responses we see that there is good agreement as the polymer transitions to its high frequency resistive response. At low frequencies there is some discrepancy in the slope of the capacitive response, however, this is attributed to the dc resistance of the transducer which is not accounted for in the transport equation. This also accounts for the phase behavior at low frequencies as the experimental data levels off near $-60^{\circ}$. Below $1 \mathrm{~Hz}$ it is observed that the phase actually increases to $0^{\circ}$ in response to the dc resistance. While the model does not capture this low frequency behavior, it is very effective in predicting the transition region of the impedance response as the polymer becomes less capacitive and exhibits a more resistive behavior.

\section{B. Impedance response as a function of diluent viscosity}

The second series of experiments considered in this work revisit the viscosity studies of Farinholt and Leo. ${ }^{15}$ Using varying concentrations of a glycerol-water mixture we obtain viscosity levels that range from 1 to $265 \mathrm{cp}$. Samples were driven with a random excitation signal while the impedance response was measured using a Tektronix 2630 Fourier analyzer. The transconductance amplifier designed by Newbury $^{47}$ was again used to condition and measure the voltage and current signals being sent to the test sample. The impedance response for the five viscosity levels are shown in Fig. 14(a). This figure illustrates the highly resistive nature that results for diluents with increasing viscosity. Relative to the model, this type of behavior is expected to have the greatest influence on the diffusion coefficient based upon the trends established in the parametric studies of Sec. VI. The model predictions for the viscosity response are shown in Fig. 14(b) and the corresponding model parameters used in each prediction are presented in Table V. In addition to the standard model parameters $T, d$, and $\kappa$, a gain factor $g$ is introduced and associated with the electrode area of the IPT transducer. This gain factor is introduced to account for variations in the electrode conductivity that result from the polymer being imbibed with different concentrations of glyc-

TABLE V. Model parameters used to simulate impedance as a function of viscosity.

\begin{tabular}{cccccc}
\hline \hline $\begin{array}{c}\text { Glycerol content } \\
(\%)\end{array}$ & $\begin{array}{c}\text { Viscosity } \\
(\mathrm{cp})\end{array}$ & $\begin{array}{c}\text { Temperature } \\
T(\mathrm{~K})\end{array}$ & $\begin{array}{c}\text { Electric permittivity } \\
\kappa(\mathrm{F} / \mathrm{m})\end{array}$ & $\begin{array}{c}\text { Diffusion coefficient } \\
\left(\mathrm{m}^{2} / \mathrm{s}\right)\end{array}$ & Gain $g$ \\
\hline 0 & 1.01 & 293 & $0.30 \times 10^{-2}$ & $13.00 \times 10^{-11}$ & 0.475 \\
60 & 10.68 & 293 & $1.00 \times 10^{-2}$ & $9.75 \times 10^{-11}$ & 0.114 \\
70 & 23.04 & 293 & $1.20 \times 10^{-2}$ & $7.80 \times 10^{-11}$ & 0.076 \\
80 & 59.90 & 293 & $1.20 \times 10^{-2}$ & $3.90 \times 10^{-11}$ & 0.067 \\
90 & 265.98 & 293 & $1.40 \times 10^{-2}$ & $1.30 \times 10^{-11}$ & 0.038 \\
\hline \hline
\end{tabular}




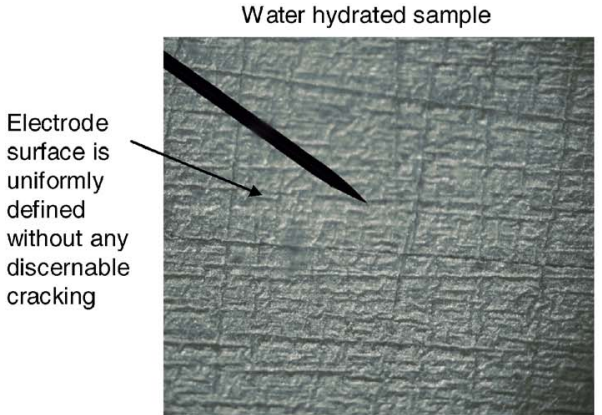

(a)

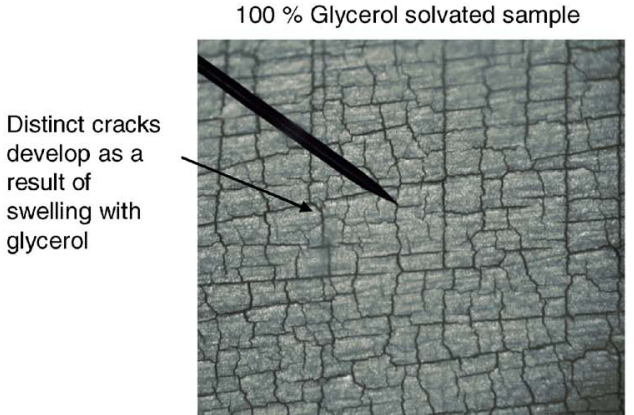

(b)
FIG. 15. (Color online) Magnified images of the surface electrode for samples solvated in $100 \%$ water (a) and $100 \%$ glycerol (b). The base line water-based sample shows a uniform electrode surface whereas the glycerol sample illustrates distinct cracking in the surface electrode as a result of membrane swelling. erol. As in the experimental work of Farinholt and Leo, ${ }^{15}$ it was visually apparent during testing that higher glycerol contents produce thin films of glycerol which coated the IPT's electrodes. Additionally, the higher glycerol concentrations were seen to induce more swelling in the membrane, producing stress on the outer electrodes of the test sample. Upon closer examination of the electrode surface, a noticeable degree of cracking was seen in the electrode, as shown in Fig. 15. From visual inspection it can be seen that electrode cracking becomes more severe at higher glycerol concentrations, a feature that is expected to greatly affect the electrode conductivity. To account for this variability a gain factor $g$ is introduced to account for unmodeled effects in the surface conductivity associated with using a one-dimensional model to predict the current response of a three-dimensional transducer.

Since the experiments were all conducted at room temperature, $T$ was set to be $293 \mathrm{~K}$ in each of the simulations, leaving the tuning parameters $\kappa, d$, and $g$. Looking initially at the experimental response of Fig. 14(a), we see that there is significant change in the resistive nature of the transducer with increasing diluent viscosity. Because of this we expect a significant variation in the polymer's diffusion coefficient at higher viscosity levels. We also see a shift in the magnitude of the low frequency response (near $1.2 \mathrm{~Hz}$ ) which is a characteristic feature of the material's capacitive response, indicating an expected variation in the polymer's effective permittivity as well. Relating the trends in the response of Fig. 14(a) with the parametric study of Sec. VI, we expect a general decrease in $d$ and an increase in $\kappa$ to correspond with changes in increased diluent viscosity. Looking at the tuning parameters of Table $\mathrm{V}$ we see that this is indeed the scaling trend exhibited by the material parameters $d$ and $\kappa$. We also note that there is a steady decline in the gain factor $g$ as the diluent viscosity increases. This trend is attributed to higher glycerol concentrations within the polymer and the diminished performance of the transducer's electrode. When this degradation of the electrode is taken into consideration it is reasonable to expect the overall conductivity of the electrode to decrease and for the measured current to decline as well. This is precisely the effect seen in the results of Fig. 14(b) as the polymer becomes much more resistive at higher glycerol concentrations. This trend is also evident in the gain factor $g$ of Table V. As the diluent viscosity increases the resultant current must decline to account for deterioration in the ionic polymer transducer's electrodes. Once all of these factors are applied to the modeling approach, we see that the impedance model can effectively predict the IPT's performance for different glycerol concentrations and viscosities. Additionally, the overall gain factor provides some accountability for changes in the properties of the external electrode.

\section{Impedance response as a function of pretension}

The third set of experiments considered in this study focus on the IPT's impedance response under pretension. The fixture of Fig. 16(a) was built to apply a longitudinal preload to the IPT during impedance measurements, relying on four parallel springs (individual spring constants: 2325 $\mathrm{N} / \mathrm{m}$ ) to apply tension to the test sample. Three levels of tension were applied to the test sample, corresponding to loads of 27.93, 102.32, and 152.12 N. A Tektronix 2630 Fourier analyzer and transconductance amplifier were again used for data acquisition.

The impedance response for these three pretension levels are presented in Fig. 16(b). The trend in these data indicates that the polymer becomes more resistive as a function of the applied tension. At low frequencies the response shows very little variation between different test conditions, indicating that the capacitive nature of the IPT is relatively invariant to change over the range of preloads considered in this study. From these trends we conclude that pretension has a predominant effect on the diffusion properties of the IPT. Since the capacitive nature does not exhibit much variation, the effective permittivity is not expected to vary greatly with longitudinal loading. Using these trends as guidelines, we simulate the polymer's predicted response using the values tabulated in Table VI. These predictions are compared to the experimental results in Fig. 16(c). From this figure we see that there is good agreement with the high frequency response, however, some variation is evident in the low frequency slope, again attributed to the dc resistance in the test sample which is not addressed in the transport model. If we now look to the model parameters used in this simulation, we see that $T, \kappa$, and the gain factor $g$ remain very consistent throughout the analysis, making the diffusion coefficient $d$ the dominant tuning parameter. In this set of experiments an overall gain factor $g$ is again introduced to account for mechanical cracking in the electrode that results from the initial pretensioning of the sample. This parameter is tuned according to the lowest level of applied tension and held fixed for the subsequent tests at 102.32 and $152.12 \mathrm{~N}$. 

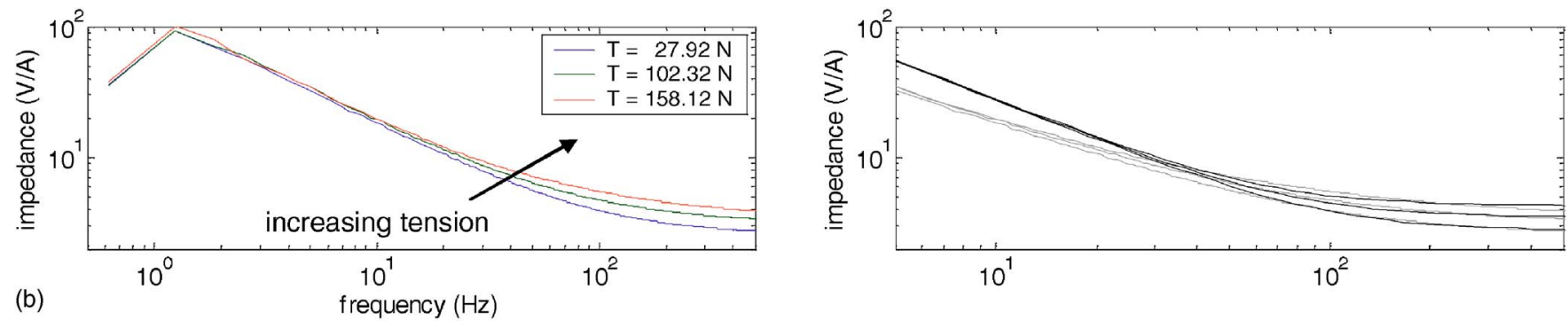

(a)
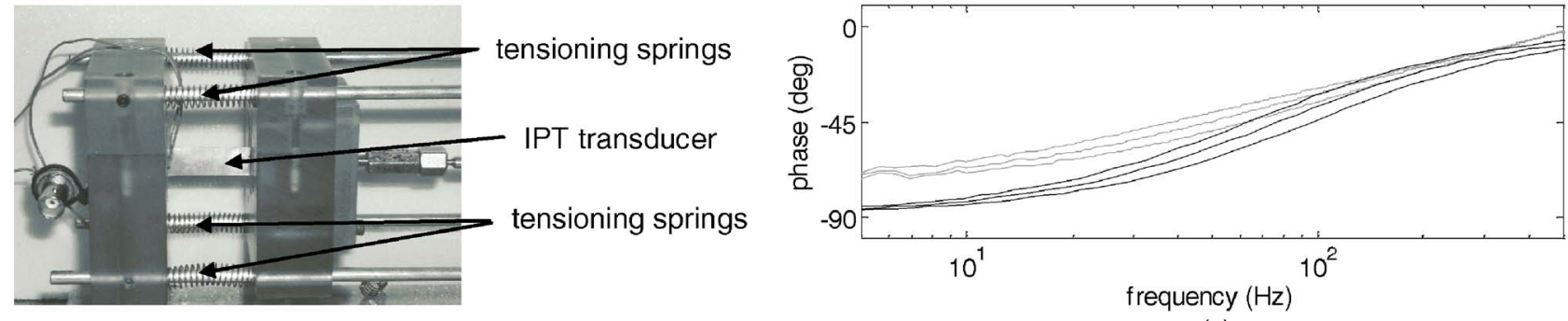

(c)

FIG. 16. (Color online) Impedance response of an IPT as a function of pretension, ranging from 27.92 to $152.12 \mathrm{~N}$ of applied tension. Plot (a) presents the results measured directly from the experiment while (b) presents a comparison with model predictions. In the comparison of (b) the gray curves correspond to experimental data and black curves correspond to model predictions.

From the parametric study of Sec. VI we note that the permittivity term is only effective in scaling the capacitive component of the IPT response, whereas the variable gain factor $g$ would simply produce shifts in the magnitude of the entire impedance response. Since the low frequency component of the measured data in Fig. 16 shows only minimal variation, neither of these parameters are suitable for describing the observed behavior. Based upon this analysis we conclude that the ionic polymer's impedance response is dominated by the diffusion coefficient in the case of longitudinal preload. Physically this implies that stress applied orthogonal to the thickness has an effect on the internal structure of the polymer which impedes the motion of ions through the polymer thickness. One explanation for this effect comes from the slight thinning expected in the width and thickness directions due to the applied longitudinal load. Intuitively this thinning would deform the internal structure of the polymer, distorting ion clusters as well as ion transport channels. Currently this is a heuristic explanation for the observed phenomena, however, future work may provide morphological insight into the polymer's structure that could be used in the fundamental design of new ionomer membranes.

\section{SUMMARY AND CONCLUSIONS}

This article presents a study of the electrical impedance response of the ionic polymer transducer. An analytical

TABLE VI. Model parameters used to simulate impedance as a function of temperature.

\begin{tabular}{ccc}
\hline \hline $\begin{array}{c}\text { Temperature } \\
T\left({ }^{\circ} \mathrm{C}\right)\end{array}$ & $\begin{array}{c}\text { Electric permittivity } \\
\kappa(\mathrm{F} / \mathrm{m})\end{array}$ & $\begin{array}{c}\text { Diffusion coefficient } \\
d\left(\mathrm{~m}^{2} / \mathrm{s}\right)\end{array}$ \\
\hline 0 & $2.26 \times 10^{-2}$ & $0.694 \times 10^{-9}$ \\
25 & $2.63 \times 10^{-2}$ & $2.133 \times 10^{-9}$ \\
40 & $2.82 \times 10^{-2}$ & $3.413 \times 10^{-9}$ \\
\hline \hline
\end{tabular}

model is proposed, based upon basic transport and field equations, describing the internal potential and charge density development within the transducer as it is subjected to an external voltage. A constant excitation is initially considered from which steady-state profiles are developed and conclusions are drawn regarding the minimum voltage needed to deplete cations near the anode surface of the IPT. This voltage is seen to be dependent upon temperature only and agrees with the predictions that can be drawn from other modeling approaches. ${ }^{26,28}$ The model is also extended to consider the effect of a harmonic voltage excitation and how this influences the saturation effect near the anode. It is determined that the minimum saturation voltage is directly related to the driving frequency, requiring higher voltages to deplete the anode region when driven at higher frequencies. In addition to the saturation study the internal charge density is used to develop an expression for the isothermal transient ionic current, which is used to estimate the surface current that would develop for given applied voltage. A sine dwell approach is used to sweep through a range of driving frequencies to generate an electrical impedance profile for the ionic polymer transducer.

Following the model development a series of analytical and experimental studies are presented to validate the proposed model's effectiveness. A parametric study was conducted to identify the effects that diffusion $d$, electrical permittivity $\kappa$, and temperature $T$ have on the transducer's characteristic impedance. Results indicate that the IPT's high frequency resistance is most affected by the ion diffusion parameter $d$. Increases in the diffusion coefficient effectively decrease the ionomer's high frequency resistance while also increasing the time constant $\lambda$. The electric permittivity $\kappa$ demonstrates an inverse effect on the IPT's impedance. Increasing values of $\kappa$ result in an increased low frequency impedance while lowering the system's characteristic time $\lambda$. Temperature effects showed an interesting response due to 
it's coupled influence with the diffusion coefficient $d$. When all other coefficients are held constant and temperature is the only variable of interest, the polymer's impedance scales in the same manner as it did with variations in $\kappa$. From experimental testing it is seen that this prediction counters the physical data that have been measured for the IPT. To address this discrepancy, the results of Yeager et al. ${ }^{41,42}$ were used to incorporate the temperature dependent variations in the diffusion coefficient $d$. These results demonstrated that the changes in $d$ outweighed those of $T$, causing the high frequency impedance of the model to decrease with temperature, a result that agrees with experimental studies.

The series of experimental studies conducted in this research also support and validate the proposed model. These experimental studies were designed to investigate three variations in the physical ionomer: (1) ambient temperature, (2) diluent viscosity, and (3) pretension applied in the longitudinal direction of the polymer. In each case, the model could accurately predict the resulting change in the transducer's response, offering insight into how changes in the effective permittivity and diffusion properties affect the ionic polymer membrane. For test conditions where the transducer experienced damage in the surface electrodes, a gain factor was introduced to account for decreased electrical conductivity due to excessive cracking. In the temperature study, parameters in the impedance model were tuned to the room temperature response. Then, temperature values were tuned in accordance with experimental conditions and the diffusion coefficient was scaled through previously published results of Yeager et al. ${ }^{41,42}$ These terms were then applied to the model to predict the response at temperatures above and below the room temperature base line. These results, coupled with those of the diluent and pretension studies, indicate very good correlations between the predicted response and measured data, while also supporting the overall validity of this electrical impedance model of the ionic polymer transducer.

\section{ACKNOWLEDGMENTS}

The authors would like to acknowledge the Army Research Office who has provided major funding through the National Defense Science and Engineering Graduate Fellowship program. This research has also been conducted in collaboration with the U.S. Army Research Laboratory and the U.S. Army Research Office under Contract/Grant No. DAAD19-02-1-0275 Macromolecular Architecture for Performance (MAP) MURI, and we would like to acknowledge their support.

\footnotetext{
${ }^{1}$ A. Katchalsky, Experientia 5, 319 (1949).

${ }^{2}$ W. Kuhn, Experientia 5, 318 (1949).

${ }^{3}$ W. Kuhn, B. Hargitay, A. Katchalsky, and H. Eisenberg, Nature (London) 165, 514 (1950).

${ }^{4}$ R. Hamlen, C. Kent, and S. Shafer, Nature (London) 206, 1149 (1965).

${ }^{5}$ I. Yannas and A. Grodzinsky, J. Mechanochem. Cell Motil. 2, 113 (1973).

${ }^{6}$ A. J. Grodzinsky and J. R. Melcher, IEEE Trans. Biomed. Eng. BME-23,
}

421 (1976).

${ }^{7}$ Y. Osada and M. Hasebe, Chem. Lett. 4, 1285 (1985).

${ }^{8}$ K. Oguro, Y. Kawami, and H. Takenaka, J. Micromachine Soc. 5, 27 (1992).

${ }^{9}$ K. Sadeghipour, R. Salomon, and S. Neogi, Smart Mater. Struct. 1, 172 (1992).

${ }^{10}$ D. Segalman, W. Witkowski, D. Adolf, and M. Shahinpoor, Smart Mater. Struct. 1, 95 (1992).

${ }^{11}$ B. J. Akle, M. Hickner, D. J. Leo, and J. E. McGrath, J. Mater. Sci. 40, 3715 (2005).

${ }^{12}$ S. Nemat-Nasser and Y. Wu, Proc. SPIE 5051, 245 (2003).

${ }^{13}$ M. Shahinpoor and K. J. Kim, Smart Mater. Struct. 3987, 110 (2000).

${ }^{14}$ K. Farinholt and D. Leo, Mech. Mater. 36, 421 (2004).

${ }^{15}$ K. Farinholt and D. Leo, J. Intell. Mater. Syst. Struct. 18, 677 (2007).

${ }^{16}$ M. Bennett and D. Leo, Sens. Actuators, A 115, 79 (2004).

${ }^{17}$ S. Zamani and S. Nemat-Nasser, Proc. SPIE 5385, 159 (2004).

${ }^{18}$ B. J. Akle, M. D. Bennett, and D. J. Leo, Proc. SPIE 5759, 153 (2005).

${ }^{19}$ R. Kanno, S. Tadokoro, and T. Takamori, Proceedings of the International Conference on Industrial Electronics, Control, and Instrumentation, 1995 (unpublished).

${ }^{20}$ R. Kanno, S. Tadokoro, T. Takamori, and M. Hattori, Proceedings of the IEEE International Conference on Robotics and Automation, 1996, pp. 219-225 (unpublished)

${ }^{21}$ K. M. Newbury and D. J. Leo, J. Intell. Mater. Syst. Struct. 13, 51 (2002).

${ }^{22}$ K. M. Newbury and D. J. Leo, J. Intell. Mater. Syst. Struct. 14, 333 (2003).

${ }^{23}$ K. M. Newbury and D. J. Leo, J. Intell. Mater. Syst. Struct. 14, 343 (2003).

${ }^{24}$ P. de Gennes, K. Okumura, M. Shahinpoor, and K. J. Kim, Europhys. Lett. 50, $513(2000)$

${ }^{25}$ S. Tadokoro, S. Yamagami, T. Takamori, and K. Oguro, 3987, 92 (2000).

${ }^{26}$ S. Nemat-Nasser, J. Appl. Phys. 92, 2899 (2002).

${ }^{27}$ S. Nemat-Nasser and J. Li, J. Appl. Phys. 87, 3321 (2000)

${ }^{28}$ S. Nemat-Nasser and S. Zamani, J. Appl. Phys. 100, 064310-1-18 (2006).

${ }^{29}$ Y. Xiao and K. Bhattacharya, Proc. SPIE 4329, 292 (2001).

${ }^{30}$ L. M. Weiland and D. J. Leo, Smart Mater. Struct. 13, 323 (2004).

${ }^{31}$ L. M. Weiland and D. J. Leo, J. Appl. Phys. 97, 013541 (2005).

${ }^{32}$ L. M. Weiland and D. J. Leo, J. Appl. Phys. 97, 123530 (2005).

${ }^{33}$ K. Asaka and K. Oguro, J. Electroanal. Chem. 480, 186 (2000).

${ }^{34}$ S. Nemat-Nasser and Y. Wu, Smart Mater. Struct. 15, 909 (2006).

${ }^{35} \mathrm{~K}$. Farinholt and D. Leo, Proc. SPIE 5387, 1 (2004).

${ }^{36} \mathrm{~N}$. Lakshminarayanaiah, Transport Phenomena in Membranes (Academic, New York, 1969).

${ }^{37}$ K. Farinholt, Modeling and Characterization of Ionic Polymer Transducers for Sensing and Actuation (Virginia Polytechnic Institute and State University, Blacksburg, VA, 2005).

${ }^{38}$ G. Greeuw and B. J. Hoenders, J. Appl. Phys. 55, 3371 (1984).

${ }^{39}$ M. Shahinpoor, J. Intell. Mater. Syst. Struct. 6, 307 (1995).

${ }^{40}$ J. W. Paquette, K. J. Kim, J.-D. Nam, and Y. S. Tak, J. Intell. Mater. Syst. Struct. 14, 633 (2003).

${ }^{41}$ H. Yeager and B. Kipling, J. Phys. Chem. 83, 1836 (1979).

${ }^{42}$ B. Steck and H. Yeager, Anal. Chem. 52, 1215 (1980).

${ }^{43}$ J. W. Paquette and K. J. Kim, Mater. Res. Soc. Symp. Proc. 785, D8.1 (2003).

${ }^{44}$ J. W. Paquette, K. J. Kim, and D. Kim, Proceedings of IMECE, 2003, p. 42929 (unpublished)

${ }^{45}$ J. W. Paquette, K. J. Kim, and D. Kim, Sens. Actuators, A 118, 135 (2005).

${ }^{46}$ K. Asaka, N. Fujiwara, K. Oguro, K. Onishi, and S. Sewa, J. Electroanal. Chem. 505, 24 (2001).

${ }^{47}$ K. M. Newbury, Modeling, Characterization, and Control of Ionic Polymer Transducers (Virginia Polytechnic Institute and State University, Blacksburg, VA, 2002)

${ }^{48}$ T. D. Gierke, G. E. Munn, and F. C. Wilson, J. Polym. Sci., Polym. Phys. Ed. 19, 1687 (1981).

${ }^{49}$ A. Goswami, A. Acharya, and A. K. Pandey, J. Phys. Chem. B 105, 9196 (2001). 